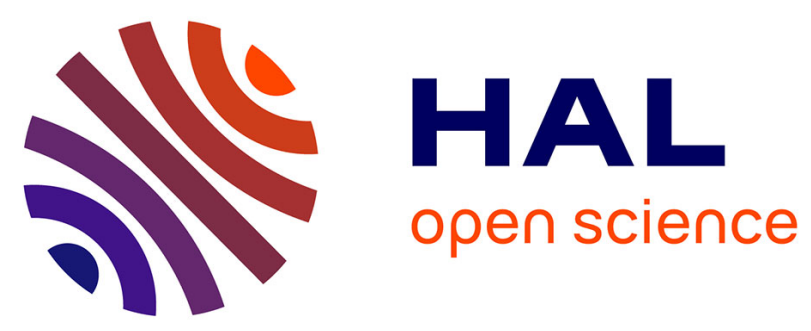

\title{
Résonance magnétique en champ de radiofréquence inhomogène - 2 e partie: Vérifications expérimentales ; mesure du coefficient de self-diffusion de $3 \mathrm{He}$
}

René Barbé, M. Leduc, F. Laloë

\section{- To cite this version:}

René Barbé, M. Leduc, F. Laloë. Résonance magnétique en champ de radiofréquence inhomogène 2 e partie: Vérifications expérimentales; mesure du coefficient de self-diffusion de $3 \mathrm{He}$. Journal de Physique, 1974, 35 (12), pp.935-951. 10.1051/jphys:019740035012093500 . jpa-00208218

\section{HAL Id: jpa-00208218 https://hal.science/jpa-00208218}

Submitted on 1 Jan 1974

HAL is a multi-disciplinary open access archive for the deposit and dissemination of scientific research documents, whether they are published or not. The documents may come from teaching and research institutions in France or abroad, or from public or private research centers.
L'archive ouverte pluridisciplinaire HAL, est destinée au dépôt et à la diffusion de documents scientifiques de niveau recherche, publiés ou non, émanant des établissements d'enseignement et de recherche français ou étrangers, des laboratoires publics ou privés. 
Classification

Physics Abstracts

$8.610-6.140$

\title{
RÉSONANCE MAGNÉTIQUE EN CHAMP DE RADIOFRÉQUENCE INHOMOGÈNE $2^{\mathrm{e}}$ partie : Vérifications expérimentales ; mesure du coefficient de self-diffusion de ${ }^{3} \mathrm{He}$
}

\author{
R. BARBÉ, M. LEDUC et F. LALOË \\ Laboratoire de Spectroscopie Hertzienne de l'E.N.S. (*), \\ 24, rue Lhomond, 75231 Paris Cedex 05, France
}

(Reçu le 24 mai 1974, révisé le 2 juillet 1974)

\begin{abstract}
Résumé. - On vérifie en détail les calculs théoriques d'un article précédent sur les formes inhabituelles de courbes de résonance en champ de radiofréquence inhomogène. Les expériences sont réalisées sur du gaz de ${ }^{3} \mathrm{He}$ orienté par pompage optique. Les différents effets prévus sont observés et étudiés (forme des deux courbes, large et étroite, différents types de déplacement des positions de la résonance, etc...). Ces expériences fournissent une mesure du coefficient de self-diffusion gazeux de ${ }^{3} \mathrm{He}$ à $300 \mathrm{~K}$ :
\end{abstract}

$$
D_{33}=1440 \pm 80 \mathrm{~cm}^{2} \mathrm{~s}^{-1} \text { à } 1 \text { torr } .
$$

\begin{abstract}
We report a detailed experimental verification of the theoretical predictions of a previous paper concerning the unusual shapes exhibited by the resonance curves when the RF field is inhomogeneous. The experiments are performed with ${ }^{3} \mathrm{He}$ gas oriented by optical pumping. The various predicted effects are observed (shape of the broad and narrow resonance curves, shifts of the resonance position arising from different origins, etc.). From these experiments one derives a measurement of the self-diffusion coefficient of gaseous ${ }^{3} \mathrm{He}$ at $300 \mathrm{~K}$ :
\end{abstract}

$$
D_{33}=1440 \pm 80 \mathrm{~cm}^{2} \mathrm{~s}^{-1} \text { at } 1 \text { torr }
$$

Introduction. - Dans un précédent article [1], nous avons présenté une étude théorique détaillée de l'effet d'un champ de radiofréquence inhomogène sur un système de spins en mouvement. Le présent article décrit une série d'expériences qui ont été entreprises dans le but de vérifier ces prévisions théoriques. Rappelons brièvement les phénomènes attendus. Dans une expérience de résonance magnétique où le champ de radiofréquence est très inhomogène, nous avons montré que les variations de l'aimantation longitudinale globale des spins donnent des formes de courbe de résonance souvent assez inhabituelles : on obtient une superposition de deux courbes de largeurs très différentes (désignées par courbe large et courbe étroite), dont les caractéristiques (forme, largeur) dépendent de la carte du champ de radiofréquence.

La courbe large s'interprète comme le résultat d'un processus de relaxation résonnante provoqué par les inhomogénéités du champ de radiofréquence;

(*) Associé au C.N.R.S. no 18 ce processus dépend essentiellement du mouvement des atomes dans le champ. Dans le cas où ce mouvement correspond à un régime de diffusion gazeuse, on peut en déduire une nouvelle méthode générale pour mesurer les coefficients de diffusion des gaz, assez simple d'emploi et qui présente l'intérêt de s'appliquer à la mesure du coefficient de self-diffusion d'un gaz.

La courbe étroite prend des formes variées selon les configurations des inhomogénéités des champs statique et de radiofréquence. En particulier, s'il existe une corrélation spatiale entre les différentes composantes de ces deux champs, des effets inhabituels sont attendus : la courbe étroite peut se présenter comme la superposition d'une courbe de dispersion et d'une courbe d'absorption, positive ou négative. De plus, on prévoit d'importants déplacements pour la position de cette résonance étroite.

Nous avons donc entrepris une vérification expérimentale de tous ces effets sur la résonance magnétique nucléaire des atomes de ${ }^{3} \mathrm{He}$ dans l'état fondamental, dont l'orientation est obtenue par pompage 
optique du gaz d'hélium soumis à une décharge faible. Toutefois, il est clair que les mêmes expériences pourraient aussi bien être répétées avec d'autres éléments. Le cas particulier de ${ }^{3} \mathrm{He}$ a été choisi pour des raisons de simple commodité et ne restreint en rien la généralité des phénomènes présentés.

Signalons que de brefs résumés des principaux résultats théoriques et expérimentaux sur ce sujet ont déjà été publiés [2], [3].

1. Principe de l'expérience. - 1.1 DisPositif expéRIMENTAL. - La méthode d'orientation nucléaire d'un gaz de ${ }^{3} \mathrm{He}$ par pompage optique a déjà fait l'objet de nombreuses publications [4], [5] ; nous ne reviendrons donc pas ici sur sa description. Rappelons simplement que la cellule de résonance étudiée contient du gaz de ${ }^{3} \mathrm{He}$ dans lequel est entretenue une décharge faible. Le processus de pompage optique indirect [6] y produit, parallèlement à une direction fixe $O z$, une certaine orientation nucléaire $M_{z}^{\mathrm{S}}$ des atomes dans l'état fondamental ${ }^{1} \mathrm{~S}_{0}$ (rappelons que l'isotope ${ }^{3} \mathrm{He}$ possède un spin nucléaire $I=\frac{1}{2}$ ); cette orientation est couramment de l'ordre de $5 \%$. Dans cette expérience, l'observable atomique détectée est, dans tous les cas, $\boldsymbol{M}_{z}$, orientation nucléaire longitudinale globale de la vapeur dans la direction $\mathrm{Oz}$ (direction qui est aussi celle du champ magnétique statique $\boldsymbol{B}_{0}$ appliqué à la cellule). La méthode de détection optique employée est décrite dans la référence [7]; elle permet de détecter $M_{z}$ avec un très bon rapport signal sur bruit.

Les cellules de résonance sont sphériques, généralement de rayon $R \simeq 3 \mathrm{~cm}$, et renferment une vapeur dont la pression $p$ est comprise entre 0,1 et 5 torr. Dans ces conditions, le libre parcours moyen $\bar{l}$ des atomes dans le gaz est faible $(\bar{l} \lesssim 3 \mathrm{~mm})$, toujours bien inférieur aux dimensions des cellules : le mouvement des atomes ${ }^{3} \mathrm{He}$ est régi par un processus de diffusion. A partir des valeurs des coefficients de diffusion de l'hélium données par d'autres auteurs [8] [9], on peut évaluer l'ordre de grandeur des temps caractérisant les modes de diffusion les plus lents pour les atomes de ${ }^{3} \mathrm{He}$ dans nos expériences; on trouve :

$$
2 \times 10^{-4}<\tau_{\mathrm{d}}<8 \times 10^{-3} \mathrm{~s}
$$

Comparons $\tau_{\mathrm{d}}$ aux temps de relaxation longitudinale $T_{1}^{0}$ et transversale $T_{2}^{0}$ de l'orientation nucléaire de ${ }^{3} \mathrm{He}$. On sait qu'à température ordinaire, dans une expérience de pompage optique de ${ }^{3} \mathrm{He}$, les temps $T_{1}^{0}$ sont très longs (couramment plusieurs dizaines de secondes), très supérieurs aux temps $T_{2}^{0}$ (qui sont déterminés par l'échange de métastabilité dans la décharge [4] [5] [6]) :

$$
0,1<T_{2}^{0}<10 \mathrm{~s} \text {. }
$$

On voit donc, en comparant (1) et (2), que la condition

$$
\tau_{\mathrm{d}} \ll T_{2}^{0}
$$

est toujours satisfaite dans nos expériences (c'est l'inégalité (A.13) de [1] qui assure que la courbe liée à la partie inhomogène est beaucoup plus large que la résonance habituelle).

Pour obtenir les champs statique et de radiofréquence inhomogènes, nous avons utilisé différents dispositifs expérimentaux que nous décrirons au fur et à mesure, selon les effets à mettre en évidence. Le plus souvent, nous avons créé les gradients de champ à l'aide de paires de bobines en position de Helmholtz connectées en opposition (bobines dites en Holtzhelm [5]). Les lignes de champ magnétique ont, dans ce cas, l'allure représentée sur la figure $1 a$ : on voit que cette configuration de champ est impaire (chaque composante du vecteur axial $\boldsymbol{B}$ est changée en son opposée dans une opération de symétrie par rapport à l'origine). Il nous est également arrivé d'utiliser des configurations de champ paires, comme c'est le cas avec une bobine unique de faible diamètre centrée sur la cellule (voir Fig. 1b). Enfin, nous nous sommes parfois servis d'un montage composé de deux fils parallèles parcourus par des courants alternatifs de même intensité et en phase : cette configuration, qui est impaire (voir Fig. 1c), présente l'intérêt de fournir un champ magnétique situé dans un plan (il n'a pas de composante parallèle à la direction des fils).
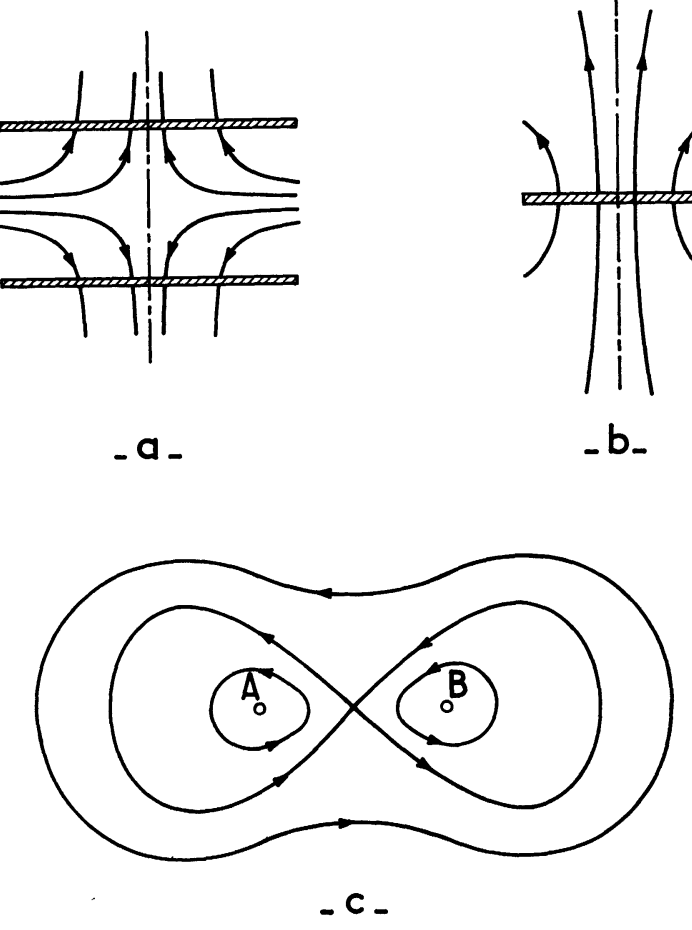

Fig. 1. - Allure des différentes configurations de champ magnétique inhomogène : $a$ ) bobines en position de Helmholtz connectées en opposition (bobines dites de Holtzhelm); b) bobine unique; c) 2 fils A et B perpendiculaires au plan de la figure et parcourus par un courant de même sens. Les configurations $(a)$ et $(b)$ donnent des champs de révolution, la configuration $(c)$ un champ parallèle au plan de la figure. 
Pour le champ statique $B_{0}$, nous avons couramment adopté la valeur : $B_{0} \simeq 10$ gauss; compte tenu du rapport gyromagnétique nucléaire de ${ }^{3} \mathrm{He}$ :

$$
\gamma=2 \pi \times 3,24 \mathrm{kHz} / \mathrm{G},
$$

ceci correspond à une fréquence de résonance $\omega / 2 \pi \simeq 32 \mathrm{kHz}$ et, en utilisant (1), on voit que :

$$
\omega \tau_{\mathrm{d}}>40 \gg 1
$$

(condition (A.15a) de [1]).

Nous avons mené les expériences de deux façons différentes : d'une part, nous avons enregistré des courbes de résonance magnétique en balayant la valeur du champ statique d'une petite quantité autour de la résonance; d'autre part, nous avons utilisé une technique de transitoires pour mesurer directement les constantes de temps de la relaxation nucléaire.

1.2 Mise EN ÉVIDENCE De LA FORME ANORMale DeS COURBES DE RÉSONANCE. - Le champ statique homogène $\boldsymbol{B}_{0}$ étant produit dans la direction $O z$, nous avons appliqué à la cellule de ${ }^{3} \mathrm{He}$ un champ radiofréquence $\boldsymbol{B}_{1}$ très inhomogène à l'aide de bobines en Holtzhelm d'axe $O x$ perpendiculaire à $O z$ (voir Fig. 2). Nous avons ainsi enregistré des courbes

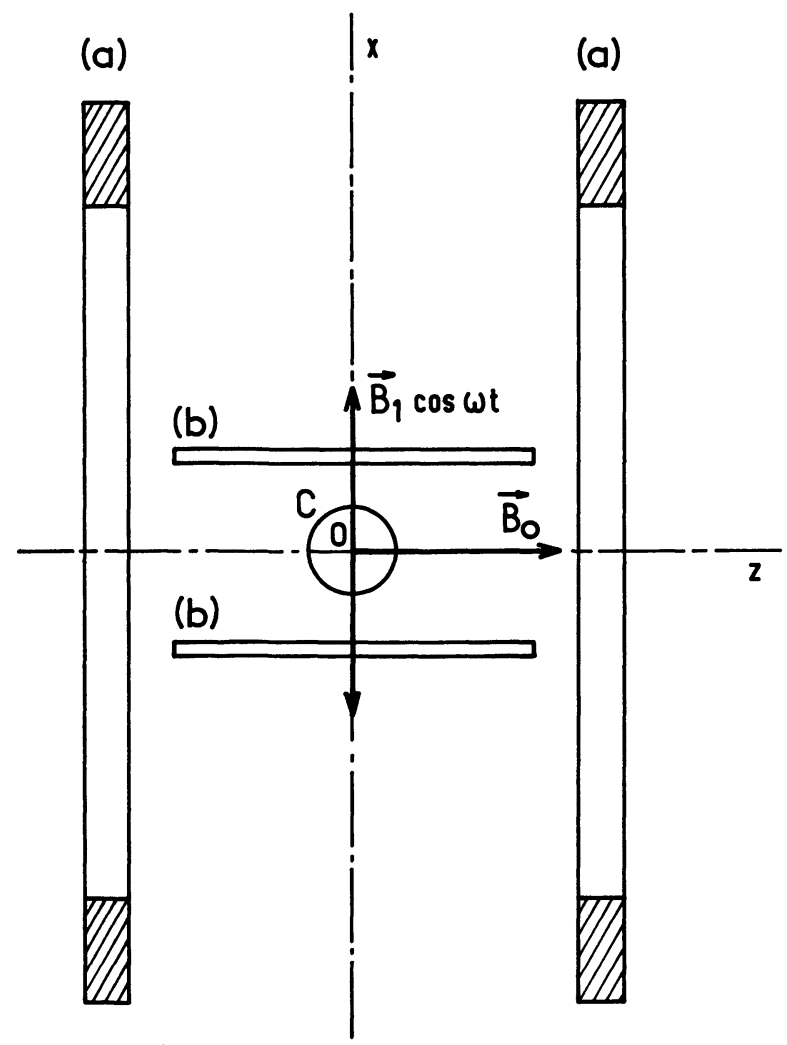

FIG. 2. - Dispositif de bobines créant les champs magnétiques : $a$ ) bobines connectées en Helmholtz créant un champ magnétique statique $\mathbf{B}_{0}$ parallèle à $\mathrm{Oz} ; b$ ) bobines connectées en Holtzhelm créant un champ magnétique oscillant $B_{1} \cos \omega t$ très inhomogène sur le volume de la cellule $C$ (les dimensions relatives de la cellule et des bobines sont respectées sur la figure).

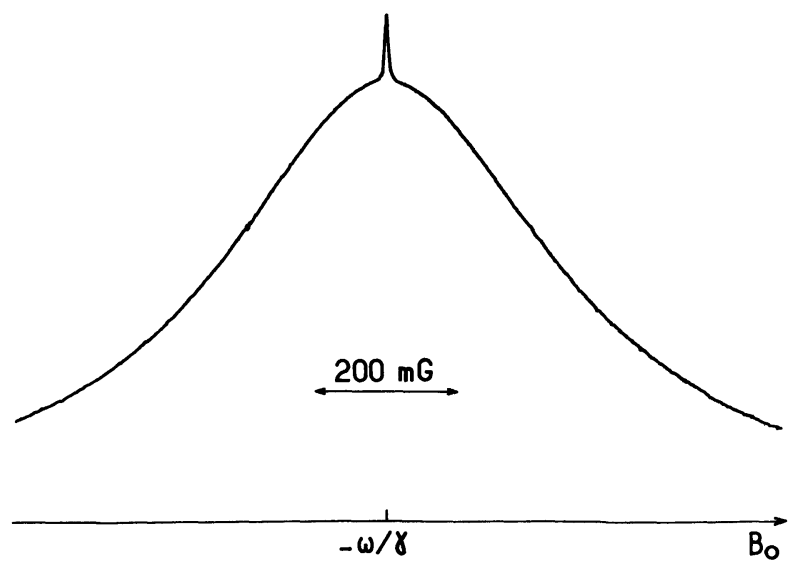

Fig. 3. - Courbe de résonance magnétique nucléaire de ${ }^{3} \mathrm{He}$ enregistrée avec un champ de radiofréquence très inhomogène (dispositif de la figure 2). Pression du gaz $: p=0,5$ torr ; rayon de la cellule : $R=3 \mathrm{~cm}$; fréquence de résonance : $\omega / 2 \pi=32 \mathrm{kHz}$.

analogues à celle de la figure 3 . Celle-ci apparaît bien comme une superposition de deux courbes de largeurs très différentes, centrées à la même valeur du champ magnétique statique, comme prévu par le modèle simple développé au § $\mathrm{A}$ de [1].

La courbe large présente une forme très sensiblement lorentzienne. Elle apparaît comme non totalement saturée. Sa largeur $\Delta B$ mesurée $(\Delta B \simeq 500 \mathrm{mG})$ est bien, en unités de pulsations $(L=\gamma \Delta B)$, du même ordre de grandeur que les valeurs de $1 / \tau_{\mathrm{d}}$ données par (1) (cf. formule (A.12) de [1]).

La courbe étroite est également une courbe d'absorption. Nous attribuons son existence au fait que le champ de radiofréquence n'est pas parfaitement inhomogène (il possède, en moyenne sur le volume de la cellule, une certaine partie homogène $\omega_{1}$ non nulle). L'observation de la courbe étroite d'absorption positive est conforme aux prévisions du $\S$ A.2 de [1]. En particulier, sa largeur $l(l$ mesuré $\simeq 1 \mathrm{mG})$ est bien du même ordre de grandeur que $1 / T_{2}^{0}$ dont les valeurs sont données par (2); (cf. formule (A.21) de [1]).

De façon à confirmer cette interprétation, nous avons cherché à annuler exactement la partie homogène $\omega_{1}$ du champ de radiofréquence sur le volume de la cellule. Pour cela, nous avons alimenté séparément chacune des deux bobines (b) de la figure 2 ; en jouant simultanément sur l'amplitude et la phase des deux tensions alternatives appliquées à ces bobines, nous avons annulé $\omega_{1}$. Dans ces conditions, on parvient à faire disparaître complètement la courbe étroite : la courbe large apparaît alors seule, comme le montre la courbe (B) de la figure 4, qui est à peu près à mi-saturation.

Dans une seconde étape, nous avons changé de $\pi$ la phase relative des deux tensions appliquées aux bobines (b) : celles-ci deviennent ainsi des bobines de Helmholtz produisant un champ de radiofréquence homogène $\omega_{1}$ intense. On enregistre alors la courbe (A) 


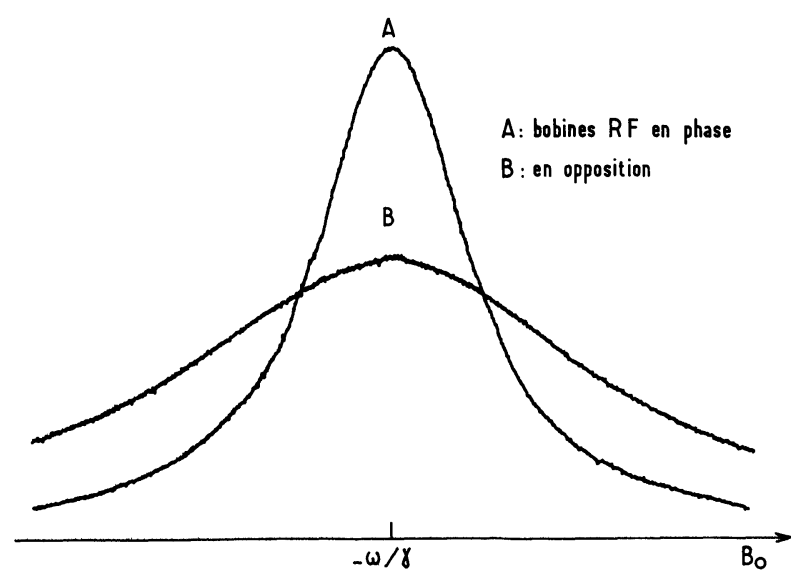

FIG. 4. - Courbes de résonance magnétique nucléaire de ${ }^{3} \mathrm{He}$ : A) les bobines $(b)$ de la figure 2 sont connectées en Helmholtz et le champ de radiofréquence est homogène; B) le champ de radiofréquence est très inhomogène; les bobines $(b)$ de la figure 2 sont connectées en Holtzhelm (la tension appliquée à chacune des bobines est la même que pour $\mathrm{A}$ ).

de la figure 4, qui est simplement une courbe de résonance lorentzienne du type habituel, mais ici très fortement saturée.

Ces deux expériences confirment donc le fait que l'existence de la courbe large de la figure 3 est bien liée à la présence d'une composante inhomogène du champ de radiofréquence.

2. Etude de la courbe large; mesure du coefficient de diffusion de l'hélium. - 2.1 COMPORTEMENT RÉSONNANT DE L'AIMANTATION LONGITUDINALE. 2.1.1 Influence de l'intensité du champ de radiofréquence. - Nous avons repris l'expérience précédente (montage de la figure 2) avec les bobines (b) de radiofréquence en Holtzhelm (le champ RF très fortement inhomogène présentant une petite partie homogène à la même fréquence). Nous avons alors fait varier son intensité : le réseau de courbes de résonance correspondant est montré sur la figure 5 .

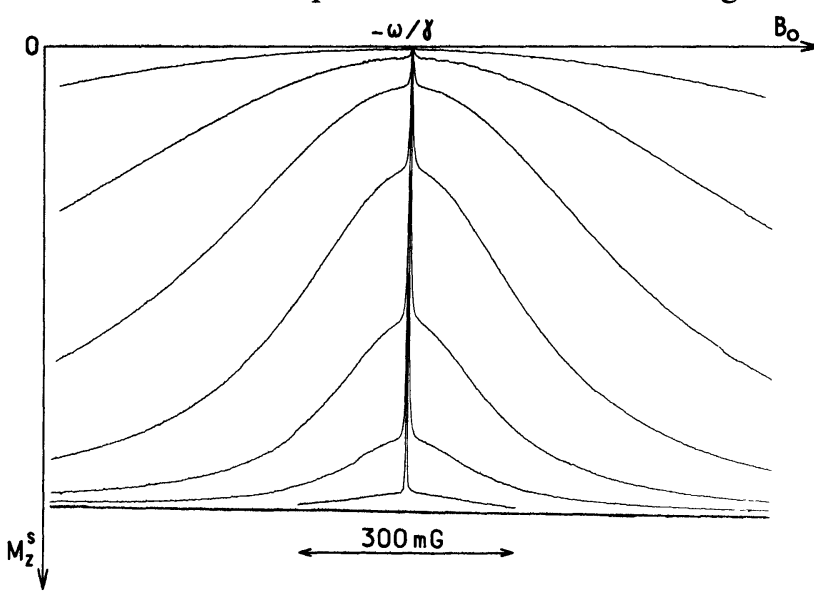

Fig. 5. - Courbes de résonance magnétique nucléaire de ${ }^{3} \mathrm{He}$ enregistrées en champ RF fortement inhomogène, pour des tensions croissantes appliquées aux bobines RF

$(p=0,39$ torr $; \omega / 2 \pi=32 \mathrm{kHz})$.

Aux faibles valeurs du champ RF la courbe fine est pratiquement seule présente; pour des valeurs plus grandes, la courbe large devient importante et finit par manger la courbe fine.
Pour l'interpréter, on doit se reporter simultanément aux formules (A.11) et (A.20) du modèle simple de [1]. A cause de l'inégalité $1 / T_{2}^{0} \ll 1 / \tau_{\mathrm{d}}$, il est clair que la courbe étroite est généralement beaucoup plus facile à saturer que la courbe large (à moins que la partie homogène du champ oscillant n'ait été très soigneusement annulée) : aux faibles intensités du champ RF, la courbe étroite apparaît donc la première. Puis, lorsque ce champ augmente, la courbe large se manifeste à son tour; elle croît, tout en s'élargissant, conformément aux prévisions de [1]. Finalement, aux fortes intensités de radiofréquence, la courbe étroite est pratiquement mangée par la courbe large.

On constate que les courbes larges enregistrées sont sensiblement lorentziennes : ceci signifie qu'avec la configuration de champ RF utilisée, l'un des modes de diffusion est beaucoup plus favorisé que les autres et apparaît seul en pratique. En fait, il est clair qu'étant donnée la symétrie impaire du champ RF créé (voir Fig. 1a), seuls des modes de diffusion correspondant à des valeurs de $l$ impaires sont susceptibles d'apparaître (voir par exemple la formule (B.31) du modèle complet de [1]). De plus, si la taille de la cellule est petite devant celle des bobines, les variations du champ peuvent, en première approximation, être considérées comme linéaires; dans ces conditions, dans la décomposition (B.31) de [1] seuls subsistent les termes correspondant au mode 3 fois dégénéré $l=n=1$; par suite le temps de diffusion qui inter-

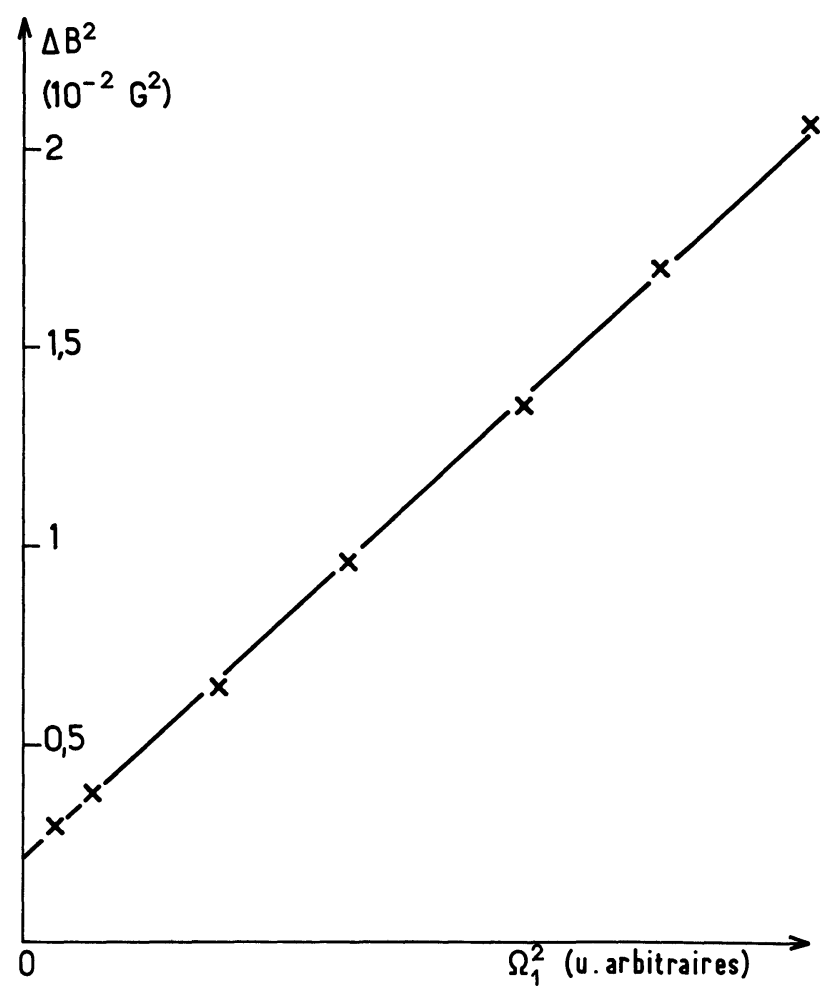

FIG. 6. - Carré des largeurs à mi-hauteur des courbes larges lorentziennes, en fonction du carré de l'intensité du champ de radiofréquence inhomogène ( $p=0,4$ torr $R=5,8 \mathrm{~cm})$. 
vient principalement dans le problème est $\tau_{11}$, donné par :

$$
\frac{1}{\tau_{11}}=\frac{4,31 D}{R^{2}}
$$

où $R$ est le rayon de la cellule sphérique et $D$ le coefficient de diffusion, inversement proportionnel à la pression $p \mathrm{du}$ gaz.

Nous avons porté sur la figure 6 le carré de la largeur $\Delta B$ de la courbe large, en fonction du carré de l'intensité du champ de radiofréquence. On trouve bien une variation linéaire, conformément à la relation (A.12) de [1] $\left(^{1}\right)$. La largeur $\Delta B^{0}$, obtenue après extrapolation à intensité nulle du champ de radiofréquence, fournit une mesure du temps de diffusion $\tau_{11}$ des atomes de ${ }^{3} \mathrm{He}$ dans la cellule. Nous reviendrons plus en détail sur ce point au $\S 2.3$ suivant.

2.1.2 Influence de la pression et de la taille de la cellule. - Nous avons fait varier la pression $p \mathrm{du}$ gaz entre 0,1 et 5 torr, et avons utilisé des cellules sphériques de trois tailles différentes. Dans chaque cas, nous avons déterminé la largeur $\Delta B^{0}$ de la courbe large extrapolée à intensité de radiofréquence nulle. Sur la figure 7 , nous avons porté en ordonnées le temps de diffusion $\tau_{11}$ déduit des mesures de $\Delta B^{0}$, en fonction de $p$. Ces résultats sont à comparer à la formule (6). On constate que, pour chaque valeur du rayon $R, \tau_{11}$ est bien proportionnel à $p$. On voit aussi sur la figure 7 que, pour une valeur donnée de $p$, $\tau_{11}$ est sensiblement proportionnel à $R^{2}$. En d'autres termes, les valeurs de $D$ déduites des pentes de chacune des trois droites de la figure 7 , correspondant à trois rayons $R$ différents, sont compatibles entre elles; nous discuterons la précision de ces mesures au $\S 2.3$. Ces résultats confirment sans ambiguïté le rôle joué par les processus de diffusion dans le problème étudié.

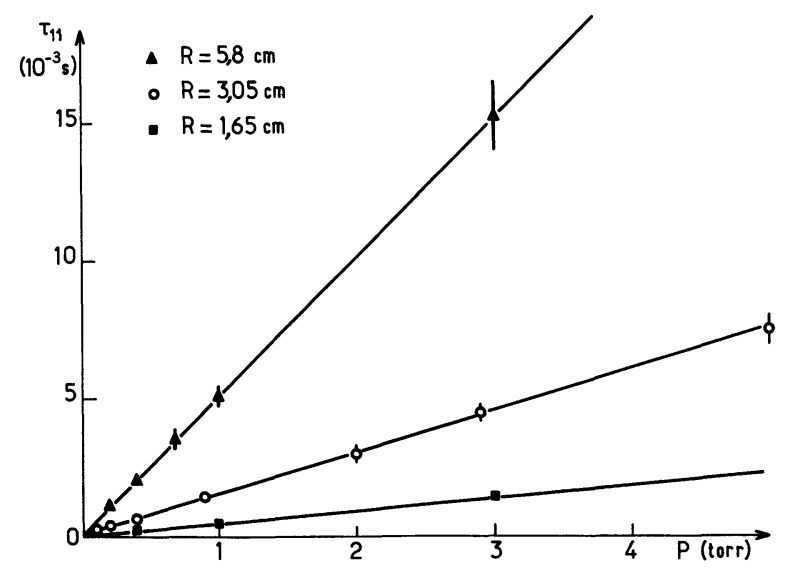

FIG. 7. - Temps de diffusion $\tau_{11}$ déduit de la largeur $\Delta B^{0}$ des courbes larges (après extrapolation à $\Omega_{1}=0$ ). $p$ : pression de ${ }^{3} \mathrm{He}$ dans la cellule. Les trois droites correspondent à 3 valeurs différentes du rayon $R$ de la cellule. Leurs pentes sont bien proportionnelles à $R^{2}$.

( $\left.{ }^{1}\right)$ Comme un seul mode de diffusion intervient ici, on peut se contenter des résultats théoriques du modèle simple ( $\$ \mathrm{~A}$ de [1]).
2.1.3 Autres modes de diffusion. - Nous avons vu au $\S 2.1 .1$ qu'avec les bobines (b) de la figure 2 en Holtzhelm, seuls les modes de diffusion $l=1$ intervenaient dans le problème. Nous avons ensuite cherché à fabriquer des configurations de champ radiofréquence différentes et plus complexes, de façon à faire apparaître d'autres modes de diffusion. $\mathrm{Si}$ plusieurs modes sont présents simultanément, le modèle simple du $\S \mathrm{A}$ de [1] n'est plus suffisant; il faut utiliser les résultats du $\S \mathrm{B}$, et en particulier reporter l'expression (B.34) dans (C.1) pour obtenir les variations de l'aimantation longitudinale; celles-ci ne correspondent plus à une seule courbe lorentzienne, mais à une superposition de telles courbes de largeurs différentes.

Expérimentalement, nous avons bien mis ce fait en évidence, par exemple avec une bobine de radiofréquence unique de petite taille centrée sur la cellule (voir Fig. 1b). La symétrie paire du champ ainsi créé entraîne que les modes de $l$ pairs ont une importance prépondérante. En fait, les modes $l=2$ et $l=4$ interviennent avec des poids du même ordre de grandeur dans nos conditions expérimentales. On enregistre alors des courbes larges analogues à celle de la figure 8 : celle-ci est visiblement non lorentzienne; sa décomposition en deux courbes de Lorentz est visible sur la figure 8 (la courbe ne comporte pas de résonance fine, car l'intensité du champ RF est assez forte pour saturer les résonances larges liées à la partie inhomogène de ce champ). Cette étude des différents modes de diffusion sera reprise avec plus de détail au $\S 2.2 .3$.

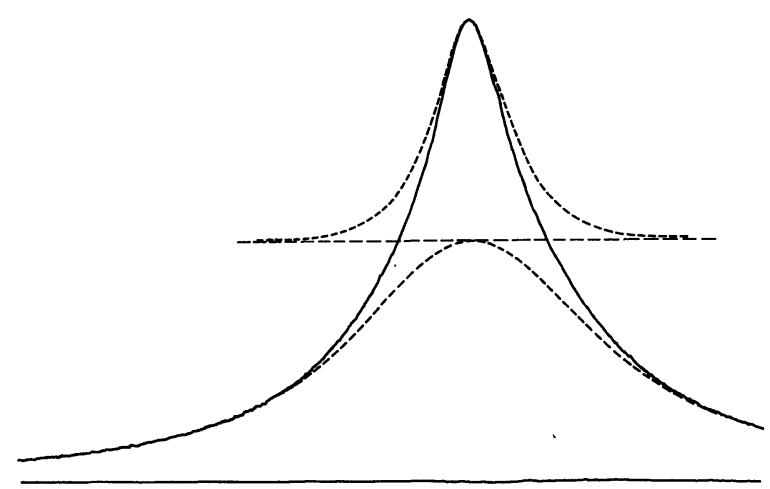

Fig. 8. - Courbe de résonance magnétique nucléaire de ${ }^{3} \mathrm{He}$ enregistrée avec une configuration particulière du champ de radiofréquence inhomogène, créé par une bobine unique centrée sur la cellule (champ pair). On a indiqué en pointillés la décomposition en deux courbes de Lorentz, qui correspondent aux modes de diffusion pairs $l=2, n=1$ et $l=4, n=1$.

\section{2 Probabilité RÉSONNANTE DE RELAXATION LON-} GITUDINALE. - Nous avons mené une étude parallèle à celle du $\S 2.1$ par une autre méthode, qui consiste à mesurer directement le temps de relaxation $T_{1}$ de l'aimantation longitudinale $M_{z}$ et non plus la valeur stationnaire $M_{z}^{S}$ de cette aimantation. La technique employée est celle des transitoires de pompage optique : à partir d'un instant initial où la vapeur possède 
de l'orientation $M_{z}^{\mathbf{S}}$, on applique brusquement le champ de radiofréquence inhomogène, tout en coupant simultanément le faisceau lumineux de pompage ; on enregistre alors les variations de $M_{z}$ en fonction du temps; $M_{z}$ décroît exponentiellement vers une valeur limite nulle, avec une constante de temps juste égale à $T_{1}$. Ces mesures peuvent être répétées à différentes valeurs du champ statique $B_{0}$ : on a ainsi une méthode d'étude de la résonance qui est en quelque sorte la transformée de Fourier de la méthode par enregistrement des courbes de résonance magnétique.

2.2.1 Influence de l'intensité des champs magnétiques. - Le champ de radiofréquence est dans cette expérience produit par les bobines (b) en Holtzhelm de la figure 2. Pour une valeur fixe de la fréquence et de l'intensité de ce champ de radiofréquence, nous avons enregistré une série de transitoires correspondant à des valeurs différentes du champ statique $B_{0}$, s'éloignant de plus en plus de la valeur de résonance $\left(B_{0}=-\omega / \gamma\right)$. Ces signaux sont visibles sur la figure 9. On vérifie bien que la décroissance de $M_{z}$

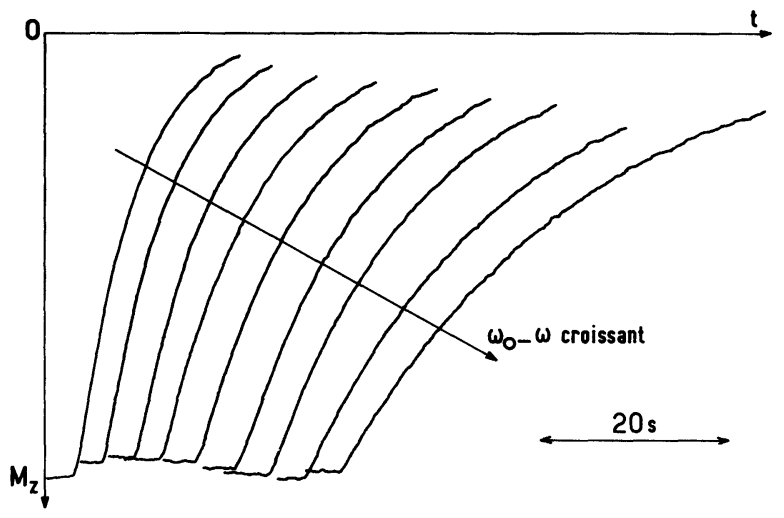

FIG. 9. - Signaux transitoires donnant la décroissance exponentielle de l'orientation nucléaire longitudinale $M_{z}$ de ${ }^{3} \mathrm{He}$ en fonction du temps, lorsqu'on applique le champ de radiofréquence inhomogène (en coupant simultanément le faisceau de pompage). Les différentes constantes de temps correspondent à un écart croissant des valeurs de $B_{0}$ par rapport à la valeur de résonance $-\omega / \gamma$.

est exponentielle pour chaque valeur de $B_{0}$. La constante de temps de l'exponentielle s'allonge quand $B_{0}$ s'écarte de la résonance, conformément à la formule (A.6) de [1]. La transitoire la plus lentement amortie de la figure 9 correspond à l'évolution de $M_{z}$ quand on coupe le faisceau de pompage sans brancher le champ de radiofréquence inhomogène : elle donne une mesure directe du temps de relaxation $T_{1}^{0}$ qui intervient dans (A.10a) de [1].

Sur la figure 10 , nous avons porté les valeurs expérimentales de la probabilité de relaxation $1 / T_{1}$ en fonction du champ statique $B_{0}$, pour plusieurs valeurs de l'intensité $\Omega_{1}$ du champ de radiofréquence inhomogène. Pour chaque valeur de $\Omega_{1}$, on constate bien que les variations de $\left(\frac{1}{T_{1}}-\frac{1}{T_{1}^{0}}\right)$ sont lorentziennes en fonction de $B_{0}$, conformément à (A.6) de [1]. On parvient à la même conclusion qu'au § 2.1.1 pour la

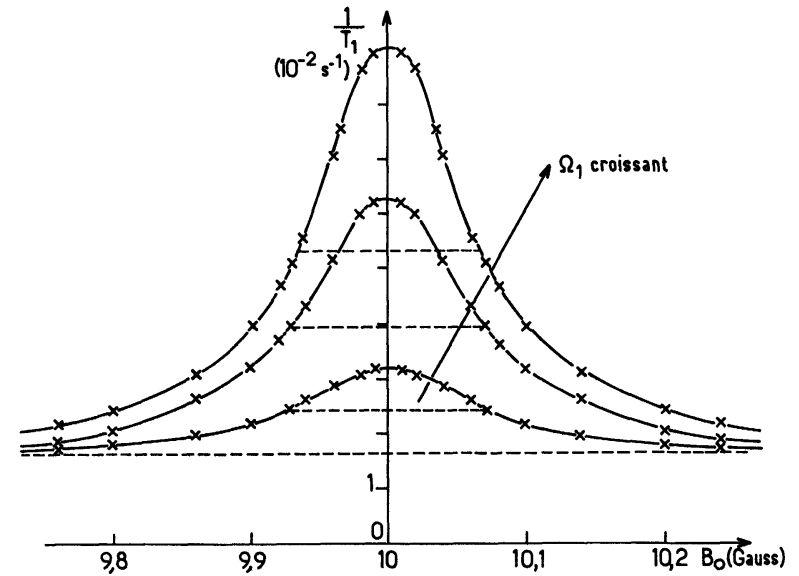

FIG. 10. - Valeurs expérimentales de la probabilité de relaxation longitudinale $1 / T_{1}$ en fonction de l'écart du champ statique $B_{0}$ par rapport à la valeur de résonance $-\omega / \gamma$. Les trois courbes correspondent à des valeurs croissantes de l'intensité $\Omega_{1} / \gamma$ du champ de radiofréquence inhomogène $(p=0,5$ torr $; R=3 \mathrm{~cm})$. On remarque l'absence d'élargissement de ces courbes par la radiofréquence.

courbe large : seuls les modes $l=n=1$ interviennent. Un fait est cependant très remarquable ici : on voit sur la figure 10 que la largeur à mi-hauteur de ces lorentziennes est indépendante de l'intensité du champ de radiofréquence. Cette largeur est, d'après (A.6), juste égale à $1 / \tau_{11}$. La connaissance d'une seule courbe de variation de $1 / T_{1}$ en fonction de $B_{0}$ suffit donc à la détermination du temps de diffusion $\tau_{11}$ : il n'est donc pas nécessaire de répéter cette expérience à différentes intensités du champ de radiofréquence. Ce fait a une importance pratique pour la mesure des coefficients de diffusion, que nous discuterons au $\S 2.3$.

On voit de plus que la valeur maximale de $1 / T_{1}$ (à résonance) augmente rapidement avec l'intensité $\Omega_{1}$ du champ de radiofréquence. D'après la formule (A.6) de [1] on prévoit que

$$
\frac{1}{T_{1}^{*}}=\left(\frac{1}{T_{1}}-\frac{1}{T_{1}^{0}}\right)
$$

varie comme $\Omega_{1}^{2}$; c'est ce que nous avons vérifié en portant sur la figure 11 la valeur de la probabilité de relaxation $1 / T_{1}$ au sommet des courbes de la figure 10 , en fonction du carré de la tension appliquée aux bornes des bobines de radiofréquence : on trouve bien une variation linéaire, l'ordonnée à l'origine étant égale à $1 / T_{1}^{0}$, probabilité de relaxation en l'absence d'inhomogénéités de champ.

A ce stade, nous avons cherché à comparer quantitativement les valeurs de $1 / T_{1}^{*}$ ainsi mesurées à celles prévues par la théorie. Il est nécessaire pour cela de se reporter aux formules du calcul complet de [1] ( $§ \mathrm{~B} .3 a$ ). Seuls les modes $l=1, n=1$ intervenant ici, la formule (B.34) se réduit (lorsque $\omega=\omega_{0}$ ) à :

$\frac{1}{T_{1}^{*}}=\left(\sum_{m}\left|c_{1 m}\right|^{2}\right) \times \frac{3}{16 \pi} \times \frac{I_{11}^{2}}{Q_{11}} \times R^{2} \times \tau_{11}$. 


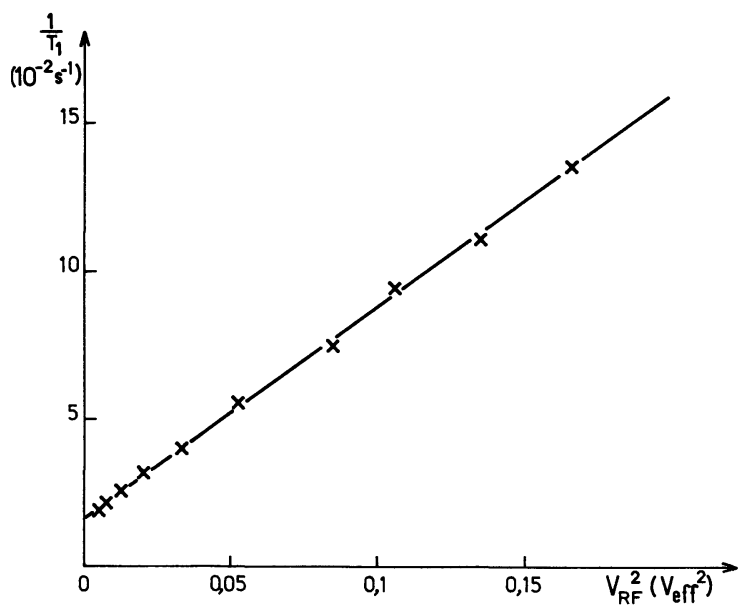

FIG. 11. - Probabilité de relaxation longitudinale $1 / T_{1}$ à résonance, en fonction du carré de l'intensité du champ de radiofréquence inhomogène $(p=0,5$ torr $; R=3 \mathrm{~cm})$.

Les intégrales de normalisation $I_{11}$ et $Q_{11}$ ont été évaluées par un calcul-machine, qui donne (voir Appendice de [1]) :

$$
I_{11}=0,1007, \quad Q_{11}=0,05123 .
$$

De plus, si l'on fait l'approximation que la configuration du champ radiofréquence $n$ 'a de composante que sur les modes $l=1$, la décomposition (B.31) donne immédiatement :

$$
\sum_{m}\left|c_{1 m}\right|^{2}=\frac{20 \pi}{3} \hat{\Omega}_{1}^{2}
$$

où $\hat{\Omega}_{1}$, exprimé en $\operatorname{rad~s}^{-1} \mathrm{~cm}^{-1}$, est l'intensité du gradient de la composante transversale tournante $\mathrm{du}$ champ créé par les bobines en Holtzhelm.

Pour l'exemple correspondant aux figures 10 et 11 ( $p=0,5$ torr), on a $R=3 \mathrm{~cm}$ et $\tau_{11}=7,35 \times 10^{-4} \mathrm{~s}$; la formule théorique (7) se réduit donc à :

$$
\frac{1}{T_{1}^{*}}=1,69 \times 10^{-3} \hat{\Omega}_{1}^{2} \quad\left(\text { avec } \hat{\Omega}_{1} \mathrm{en} \mathrm{rad} \mathrm{s}^{-1} \mathrm{~cm}^{-1}\right) .
$$

Le coefficient de cette formule est à comparer à la pente de la droite de la figure 11. Nous avons effectué un étalonnage en valeur absolue de l'intensité du champ RF à l'aide d'une petite bobine-sonde explorant la carte du champ à l'intérieur des bobines (b) en Holtzhelm, Nous avons ainsi déterminé que $\hat{\Omega}_{1}=21,5 V_{\mathbf{R F}}$ (où $V_{\mathbf{R F}}$ est la tension appliquée aux bobines en volts efficaces). La pente de la figure 11 fournit ainsi le résultat expérimental :

$$
\frac{1}{T_{1}^{*}}=0,72 V_{\mathrm{RF}}^{2}=1,55 \times 10^{-3} \hat{\Omega}_{1}^{2}
$$

qui est bien compatible avec le résultat théorique (8). L'accord obtenu peut même être considéré comme très bon, compte tenu des approximations faites pour 'le calcul (champ RF décomposable sur les modes $l=n=1$ uniquement) et des erreurs expérimentales (dues principalement à l'étalonnage du champ RF).

2.2.2 Influence de la taille de la cellule. - Nous avons fait varier le rayon de la cellule de façon à vérifier les prévisions du $\S$ B. $3 a$ de [1]. Sur la figure 12 , nous avons porté les résultats expérimentaux obtenus pour deux cellules contenant la même pression de gaz mais de rayons $R_{1}$ et $R_{2}$ différents $\left(R_{1}=2,9 \mathrm{~cm}\right.$, $R_{2}=1,5 \mathrm{~cm}$ ); les deux courbes sont relatives à une même tension appliquée sur les bobines créant le champ de radiofréquence inhomogène. Pour $l=1$,

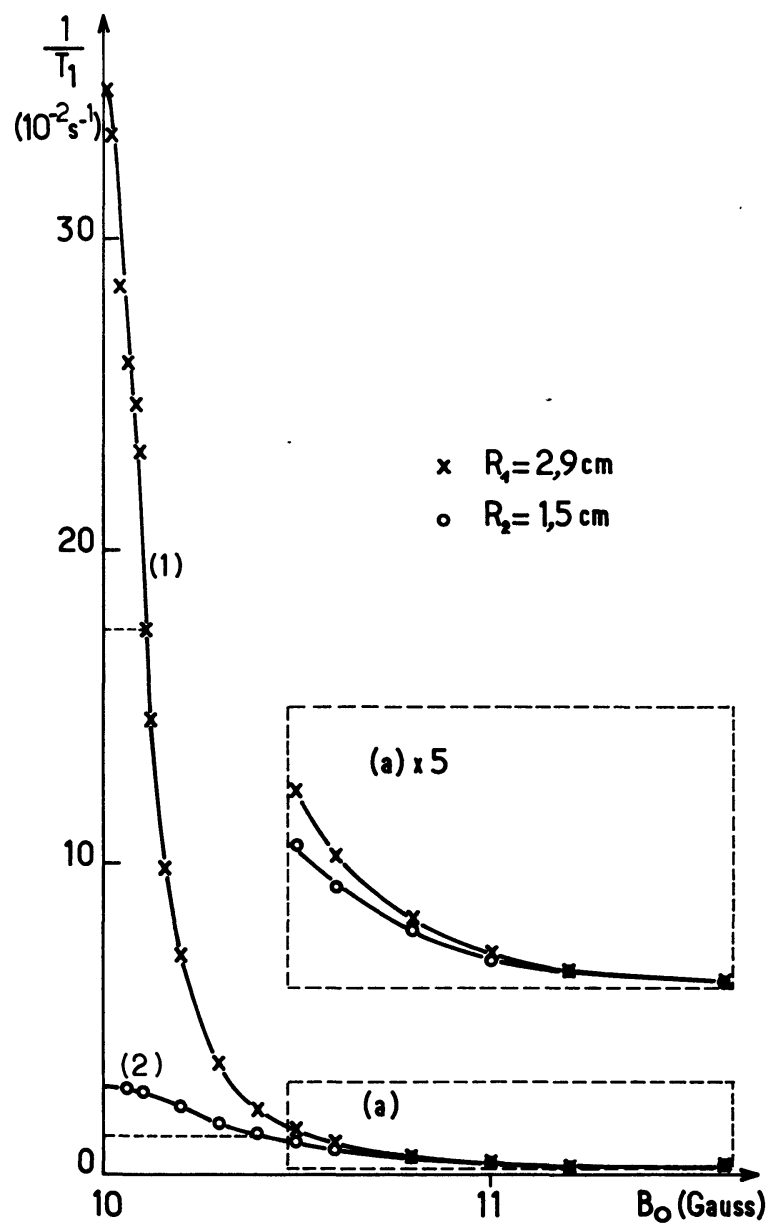

FIG. 12. - Probabilité de relaxation longitudinale $1 / T_{1}$ en fonction du champ magnétique statique $B_{0}$, pour deux valeurs $R_{1}$ et $R_{2}$ du rayon de la cellule et une même valeur de la pression ( $p=0,39$ torr) : $R_{1}=2,9 \mathrm{~cm} ; R_{2}=1,5 \mathrm{~cm}$. La partie encadrée en pointillés (a) a été reportée sur la même figure après multiplication des ordonnées par un facteur 5 .

la formule (B. $35 b)$ se réduit à $1 / T_{1}^{*}=$ Cte : loin de la résonance la relaxation est indépendante du rayon de la cellule; c'est bien ce que l'on constate sur la figure 12 , où l'on voit les courbes (1) et (2) tendre asymptotiquement l'une vers l'autre aux fortes valeurs de $B_{0}$. Par contre les formules (8) de l'Appendice de [1] et (B.35a) deviennent ici :

$$
\left\{\begin{aligned}
\tau_{11} & \propto R^{2} \\
1 / T_{1}^{*} & \propto R^{4} \text { à la résonance . }
\end{aligned}\right.
$$


Le tableau I résume la comparaison de ces prévisions théoriques aux résultats expérimentaux. On constate que l'accord est bon.

\section{TABLEAU I}

Résultats relatifs aux hauteurs et largeurs des courbes 1) et 2) de la figure 12, qui correspondent à des cellules de rayon $R_{1}=2,9 \mathrm{~cm}$ et $R_{2}=1,5 \mathrm{~cm}$ respectivement.

\begin{tabular}{l|c|c} 
& Résultats expérimentaux & Résultats théoriques \\
\hline largeur (2) & 3,6 & $\left(\frac{R_{1}}{R_{2}}\right)^{2}=3,8$ \\
\hline largeur (1) & 13,8 & $\left(\frac{R_{1}}{R_{2}}\right)^{4}=14$
\end{tabular}

2.2.3 Autres modes de diffusion. - Comme au $\S 2.1 .3$, nous avons cherché à faire apparaître des modes de diffusion plus élevés en considérant d'autres configurations de champ radiofréquence. Nous avons en particulier utilisé une bobine unique de petit diamètre $(\simeq 8 \mathrm{~cm})$, d'axe parallèle à $O z$ et déplaçable sur cet axe. Les variations de $1 / T_{1}^{*}$ en fonction de $B_{0}$ sont alors des superpositions de courbes de Lorentz; nous avons dépouillé les courbes obtenues de façon à déduire dans chaque cas la largeur et le poids de ces différentes courbes de Lorentz superposées. Nous avons ainsi mis en évidence jusqu'à 3 modes de diffusion différents intervenant dans une même expérience.

Les résultats sont schématisés sur la figure 13 . La première ligne rappelle ce que nous avons trouvé avec des bobines en Holtzhelm (champ impair) de $30 \mathrm{~cm}$ de diamètre : seul le mode $l=n=1$ apparaît.
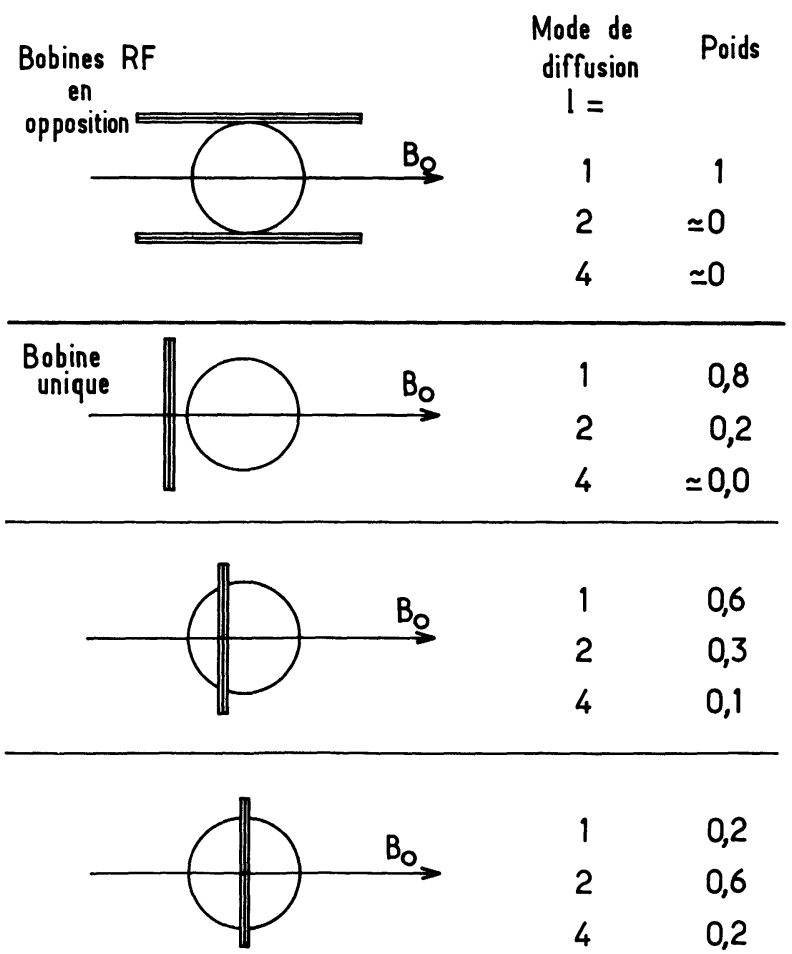

Fig. 13. - Poids des différents modes de diffusion observés par la méthode des transitoires pour différentes configurations de champ de radiofréquence inhomogène.
La dernière ligne correspond au cas où la bobine de radiofréquence est unique et centrée sur la cellule (champ assez sensiblement pair) : les modes pairs (ici $l=2, n=1$ et $l=4, n=1$ ) sont très favorisés par rapport aux modes impairs : le poids du mode $l=1$ a beaucoup diminué par rapport au cas de la première ligne (s'il n'a pas disparu totalement, c'est que le centrage de la bobine sur la cellule n'est pas géométriquement parfait, et donc que la symétrie du champ créé n'est pas complètement paire). Les deux autres lignes de la figure 13 rendent compte des résultats dans le cas intermédiaire où le champ radiofréquence a une partie paire et une partie impaire : des modes de $l$ pair et impair interviennent simultanément.

2.3 Mesure DU COEFFICIENT DE DIFFusion DE ${ }^{3} \mathrm{He}$. - Il ressort clairement de ce qui précède que, pour faire une mesure du coefficient de diffusion des atomes, on a intérêt à utiliser une configuration de champ RF inhomogène telle que le mode le plus bas $(n=l=1)$ intervienne seul. C'est ce que nous avons réalisé avec une bonne précision grâce aux bobines en position de Holtzhelm de diamètre $30 \mathrm{~cm}$. Nous avons vu que la détermination de $\tau_{11}$ peut se faire par deux méthodes différentes. D'une part, on peut enregistrer un réseau de courbes de résonance larges pour différentes intensités $\Omega_{1}$ du champ de radiofréquence; on mesure alors leur largeur à mi-hauteur, dont on extrapole la valeur à $\Omega_{1}$ nul. D'autre part, on peut utiliser la technique des transitoires de relaxation, qui fournit $\tau_{11}$ par une unique courbe correspondant à une valeur quelconque de $\Omega_{1}$. La seconde méthode apparaît en pratique plus précise que la première, car elle ne nécessite pas d'extrapolation à $\Omega_{1}=0$. Elle est d'ailleurs beaucoup plus simple d'emploi et aussi plus rapide (au moins dans le cas de ${ }^{3} \mathrm{He}$, où l'enre-

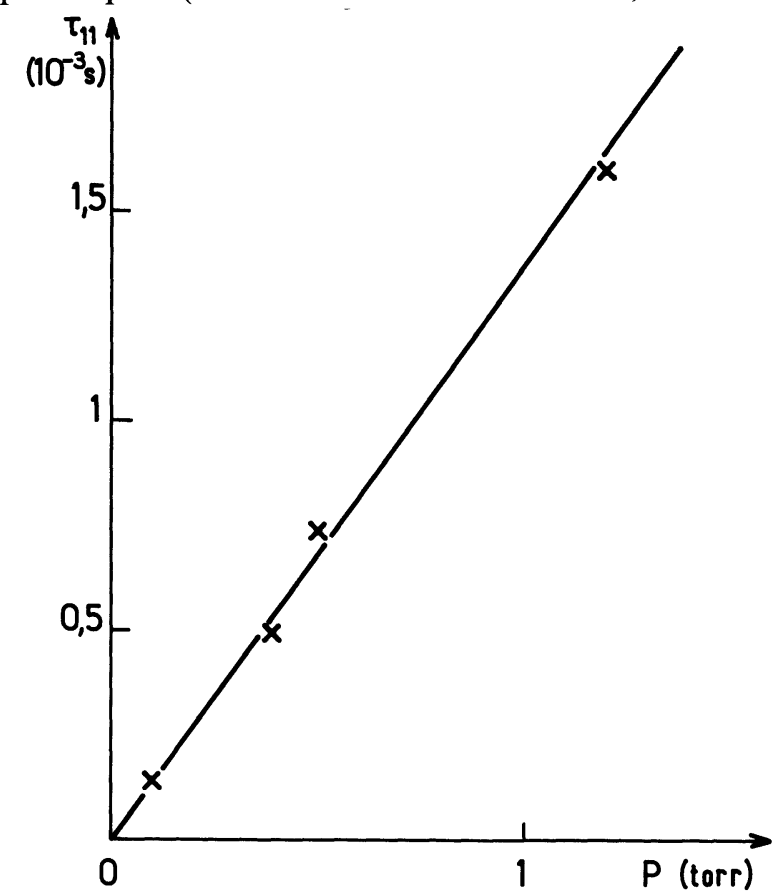

FIG. 14. - Temps de diffusion $\tau_{11}$ déduit de la mesure des temps de relaxation longitudinale. $p$ : pression de ${ }^{3} \mathrm{He} . R=2,9 \mathrm{~cm}$. 
gistrement sans distorsion des courbes de RMN est très long, étant données les grandes constantes de temps de réponse du système atomique). Finalement la mesure du coefficient de diffusion $D$ de ${ }^{3} \mathrm{He}$ que nous adoptons est celle qui est déduite de la figure 14 ; nous y avons porté les valeurs de $\tau_{11}$ trouvées par la méthode des transitoires de relaxation, en fonction de la pression d'hélium, dans des cellules de rayon $R$ constant. De la pente de la droite obtenue, on déduit à l'aide de la formule (6) :

$$
D=(1440 \pm 80) \mathrm{cm}^{2} \mathrm{~s}^{-1} \text { à } 1 \text { torr . }
$$

La barre d'erreur indiquée pour $D$ provient essentiellement de l'incertitude sur les valeurs de la pression et du rayon intérieur de la cellule, jamais parfaitement sphérique. Le temps de diffusion $\tau_{11}$ lui-même est donc déterminé avec une précision bien supérieure à celle du coefficient $D$.

$D$ est un coefficient de self-diffusion, c'est-à-dire relatif à la diffusion des atomes de ${ }^{3} \mathrm{He}$ dans l'état fondamental à travers un gaz constitué des mêmes atomes ${ }^{3} \mathrm{He}$ et à notre connaissance, ce coefficient (encore appelé $D_{33}$ ) n'avait jamais été mesuré auparavant. Cependant, il existe différents travaux antérieurs qui ont déterminé le coefficient $D_{34}$ (diffusion de l'isotope ${ }^{3} \mathrm{He}$ dans un gaz de ${ }^{4} \mathrm{He}$ ) [8] [9]. Les méthodes employées sont totalement différentes de celles décrites ici, et dans l'ensemble plus complexes : elles impliquent en particulier l'utilisation de la spectrographie de masse pour doser les deux isotopes de l'hélium après la diffusion. On peut chercher à comparer les valeurs de $D_{34}$ trouvées antérieurement à celle de $D_{33}$ que nous proposons. Pour cela nous supposerons que la section efficace de diffusion est indépendante de l'isotope d'hélium considéré. Dans ces conditions, le coefficient de diffusion $D$ est simplement proportionnel à la vitesse relative des deux atomes entrant en collision. Les vitesses atomiques sont thermiques, donc proportionnelles à $1 / \sqrt{m}$, où $m$ est la masse atomique. D'où :

et

$$
D_{34} \propto \sqrt{\frac{1}{m_{3}}+\frac{1}{m_{4}}}
$$

$$
D_{33} \propto \sqrt{\frac{2}{m_{3}}} \text {. }
$$

On en déduit que :

$$
D_{33}=D_{34} \cdot \sqrt{\frac{8}{7}} .
$$

Ceci permet de comparer les résultats de [8] [9] au nôtre. Sur le tableau II, nous avons inscrit dans les deux premières lignes de la dernière colonne les valeurs de $D_{33}$ déduites des mesures [8] et [9] à l'aide de la relation (10). Elles sont en très bon accord avec la valeur donnée par notre expérience ( $3^{e}$ ligne), compte tenu des barres d'erreur.

\begin{tabular}{|c|c|c|}
\hline Références & $\begin{array}{c}D_{34} \\
\mathrm{~cm}^{2} \mathrm{~s}^{-1} \text { à } 1 \text { torr }\end{array}$ & $\begin{array}{c}D_{33} \\
\mathrm{~cm}^{2} \mathrm{~s}^{-1} \text { à } 1 \text { torr }\end{array}$ \\
\hline- & $1310+40$ & $1400+50$ \\
\hline Mnériance & $1320 \pm 50$ & $1410 \pm 60$ \\
\hline
\end{tabular}

TABLEAU II

Mesure du coefficient de diffusion de l'hélium à la température ordinaire

La précision des différents types de mesure de $D$ est du même ordre de grandeur.

La méthode de mesure des coefficients de selfdiffusion décrite ici s'apparente par certains aspects à celle dite des échos de spin [10] [11]. Dans celle-ci on peut en effet relier l'amortissement de l'amplitude de l'écho au coefficient de diffusion du gaz, à condition de connaître la valeur absolue du gradient de champ statique appliqué. Notons que la méthode décrite ici ne requiert pas la connaissance de la valeur du gradient de champ radiofréquence utilisé, ce qui peut la rendre éventuellement plus précise que celle des échos de spin. De plus, elle ne nécessite pas l'emploi des techniques de pulses de champ. On peut envisager de l'étendre à de nombreux autres gaz, sur lesquels on sait produire la résonance magnétique. Signalons que C. Fabre et J. Dupont-Roc ont récemment observé une courbe large sur $d{ }^{87} \mathrm{Rb}$ pompé optiquement $\left({ }^{2}\right)$ tandis que Novikov et al. ont mis en évidence le même effet dans le cas de ${ }^{199} \mathrm{Hg}$ et ${ }^{201} \mathrm{Hg}$ [12], donnant ainsi l'interprétation correcte des formes de courbes anormales décrites par ces mêmes auteurs dans une publication très antérieure [13].

3. Etude de la courbe étroite; déplacements de fréquence. -3.1 DÉPLACEMENT $\delta \omega_{0}^{*}$ DE LA FRÉQUENCE DE RÉSONANCE. - 3.1.1 Mise en évidence. - Dans le $\S 2$ précédent, nous avons étudié de différentes façons l'effet d'un champ de radiofréquence inhomogène sur l'aimantation longitudinale des atomes. Nous avons plus particulièrement mis en évidence les variations résonnantes de la relaxation longitudinale $1 / T_{1}$. Mais nous avons vu, au $\S$ B.3a de [1], que l'aimantation transversale est également affectée par le champ RF inhomogène : la probabilité $1 / T_{2}$ de relaxation transversale subit aussi des variations résonnantes; de plus une certaine modification $\delta \omega_{0}^{*}$ de la précession de cette aimantation transversale est prévisible. L'expression complète de $\delta \omega_{0}^{*}$ est donnée par la formule (B.39a) de [1] dans le cas général. Dans le cas où un seul mode de diffusion intervient, cette formule se simplifie (cf. (B.39b)). L'expression (B.39b) montre bien que les variations résonnantes de $1 / T_{1}^{*}$ et de $\delta \omega_{0}^{*}$ sont deux effets intimement liés.

Un tel déplacement de la fréquence de résonance est nul à résonance $\left(B_{0}=-\omega / \gamma\right)$. Pour le mettre en évidence, nous avons dû utiliser deux champs de

(2) FABRE, C. et Dupont-Roc, J., Communication privée. 
radiofréquence distincts. Le premier, de pulsation $\omega$, est purement inhomogène (il est créé par les bobines (b) en Holtzhelm de la figure 2); son rôle est de créer le déplacement de fréquence. Le second, de pulsation $\omega^{\prime}$ différente de $\omega$, est purement homogène et beaucoup moins intense que le précédent. Il est destiné à produire une résonance-sonde (courbe étroite) qui ne soit pas située au centre de la courbe large. Le principe de la
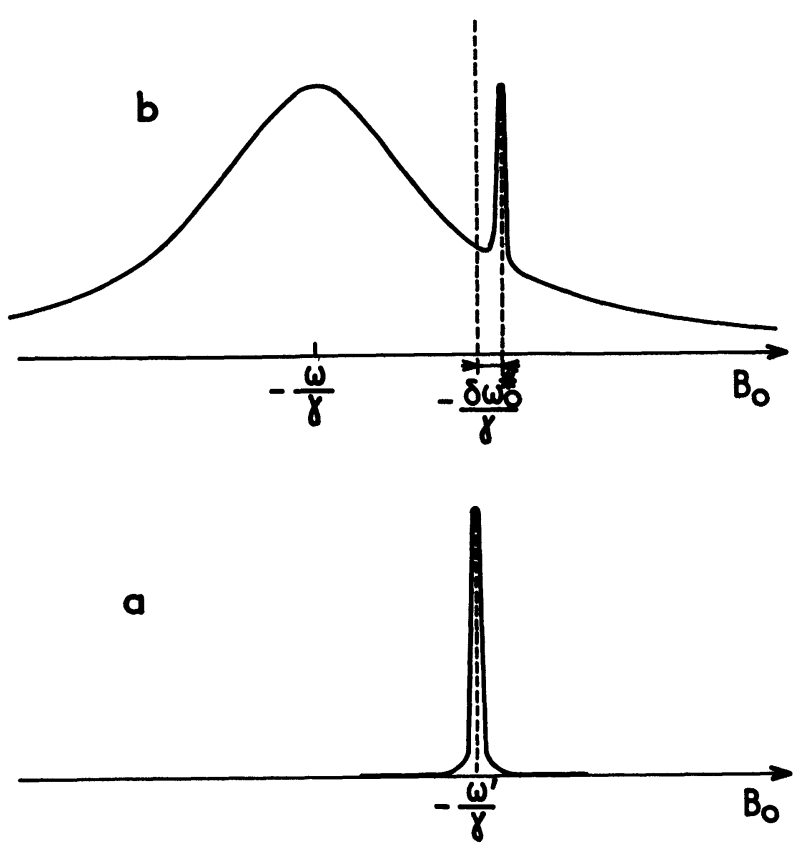

Fig. 15. - Principe de la méthode de mise en évidence du déplacement $\delta \omega_{0}^{*} / 2 \pi$ de la fréquence de résonance : $a$ ) un premier champ RF, homogène, peu intense, de fréquence $\omega^{\prime} / 2 \pi$, permet d'enregistrer une courbe de résonance fine ordinaire centrée à la valeur $-\omega^{\prime} / \gamma$ de $B_{0} ; b$ ) un deuxième champ RF, complètement inhomogène, très intense, de fréquence $\omega / 2 \pi$, est appliqué en plus du précédent. La résonance fine est déplacée d'une quantité $-\delta \omega_{0}^{*} / \gamma$ par rapport à sa position $(a)$.

mise en évidence expérimentale du déplacement $\delta \omega_{0}^{*}$ est illustré sur la figure 15 . La figure $15 a$ montre la courbe de résonance étroite attendue, à la valeur - $\omega^{\prime} / \gamma$ du champ statique $B_{0}$, lorsque le champ RF homogène est seul présent (résonance magnétique habituelle). La figure $15 b$ montre ce qui doit se produire lorsqu'on applique simultanément le champ RF inhomogène : d'une part une courbe large centrée en $B_{0}=-\omega / \gamma$; d'autre part une courbe étroite sur l'aile de cette courbe large, dont la position n'est pas la même que dans le cas de la figure $15 a$ (déplacement vers les ailes de la courbe large, d'après (B. $39 b)$ de [1]); sa largeur et sa hauteur sont d'ailleurs également modifiées.

Les résultats de l'expérience sont présentés sur la figure 16. Sur la partie inférieure ( $a$ ) apparaît la courbe de résonance étroite sans champ inhomogène (ici $\left.\omega^{\prime} / 2 \pi=172 \mathrm{kHz}\right)$; sa largeur est de quelques hertz $\left(\sim 1 / T_{2}^{0}\right.$ loin de la saturation). La partie supérieure $(b)$ de la figure 16 montre ce que devient cette résonance lorsqu'on applique le champ RF inhomogène; les deux courbes enregistrées correspondent à deux

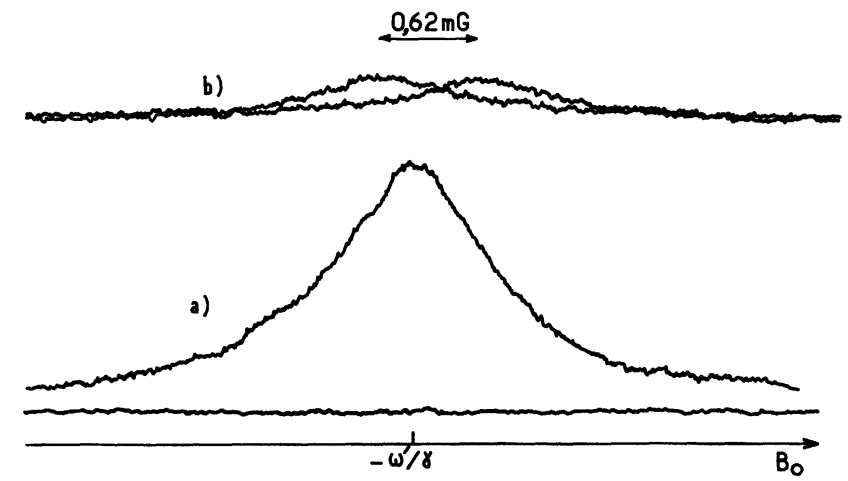

Fig. 16. - Mise en évidence expérimentale du déplacemént de fréquence $\delta \omega_{0}^{*} / 2 \pi$ dans le cas de la RMN de ${ }^{3} \mathrm{He}$. a) Courbe de résonance ordinaire en champ RF homogène $\left(\omega^{\prime} / 2 \pi=172 \mathrm{kHz}\right)$; b) un champ RF inhomogène est appliqué, en plus du précédent, à la pulsation $\omega=\omega^{\prime} \pm \Delta(\Delta / 2 \pi=30 \mathrm{kHz})$.

valeurs différentes $\omega=\omega^{\prime} \pm \Delta$ de la pulsation du champ inhomogène (ici $\Delta / 2 \pi=30 \mathrm{kHz}$ ), pour une même intensité $\Omega_{1}$ de ce champ. La valeur de $\Omega_{1}$ a été choisie aussi grande que possible ( $\delta \omega_{0}^{*}$ est en effet proportionnel à $\Omega_{1}^{2}$ ), sa limite étant évidemment imposée par le fait qu'il doit subsister une certaine orientation atomique au champ $B_{0}=-\omega^{\prime} / \gamma$, de façon à ce que les courbes de résonance de la figure $16 b$ soient encore visibles, malgré l'importance du fond continu (aile de la courbe large). Avec les valeurs de $\Delta$ choisies, on voit que la quantité $\left|\omega_{0}-\omega\right| \tau_{\mathrm{d}}$ des formules (B.39) de [1] est très grande devant 1 $\left(\Delta \tau_{\mathrm{d}} \simeq 100\right)$. La courbe large est ici très fortement élargie par le champ RF inhomogène (sa largeur est plus de 100 fois celle qu'elle aurait si elle était désaturée). Le déplacement $\pm \delta \omega_{0}^{*} / \gamma$ de la position des résonances apparaît clairement sur la figure $16 b$. On remarque que le signe de l'effet correspond aux prévisions $\left(\delta \omega_{0}^{*}>0\right.$ pour $\omega=\omega^{\prime}-\Delta$ et $\delta \omega_{0}^{*}<0$ pour $\omega=\omega^{\prime}+\Delta$, d'après (B.39b) de [1]). Le déplacement $\delta \omega_{0}^{*}$ est ici du même ordre de grandeur (quelques hertz) que la largeur de la résonance; cet effet n'est donc pas négligeable.

3.1.2 Vérifications quantitatives. - Nous avons répété des enregistrements analogues à ceux de la figure $16 b$ en faisant varier les paramètres $\Omega_{1}$ et $\Delta$, de façon à vérifier quantitativement les prévisions théoriques (B.39b) de [1]. La figure 17 montre les valeurs obtenues pour $2 \delta \omega_{0}^{*} / 2 \pi$, en fonction du carré de l'intensité $\Omega_{1}$ du champ de radiofréquence inhomogène, pour une valeur fixe de $\Delta(\Delta / 2 \pi=30 \mathrm{kHz})$. Compte tenu de la forte imprécision inhérente à la mesure de $\delta \omega_{0}^{*}$, on voit que ces résultats sont compatibles avec une droite passant par l'origine. Ceci est en accord avec la formule (B.39b) de [1].

A valeur de $\Omega_{1}$ fixée, nous avons également fait varier le paramètre $\Delta$. Pour chaque valeur de $\Delta$, nous avons simultanément mesuré par la méthode des transitoires la probabilité de relaxation

$$
\frac{1}{T_{1}^{*}}=\frac{1}{T_{1}}-\frac{1}{T_{1}^{0}}
$$




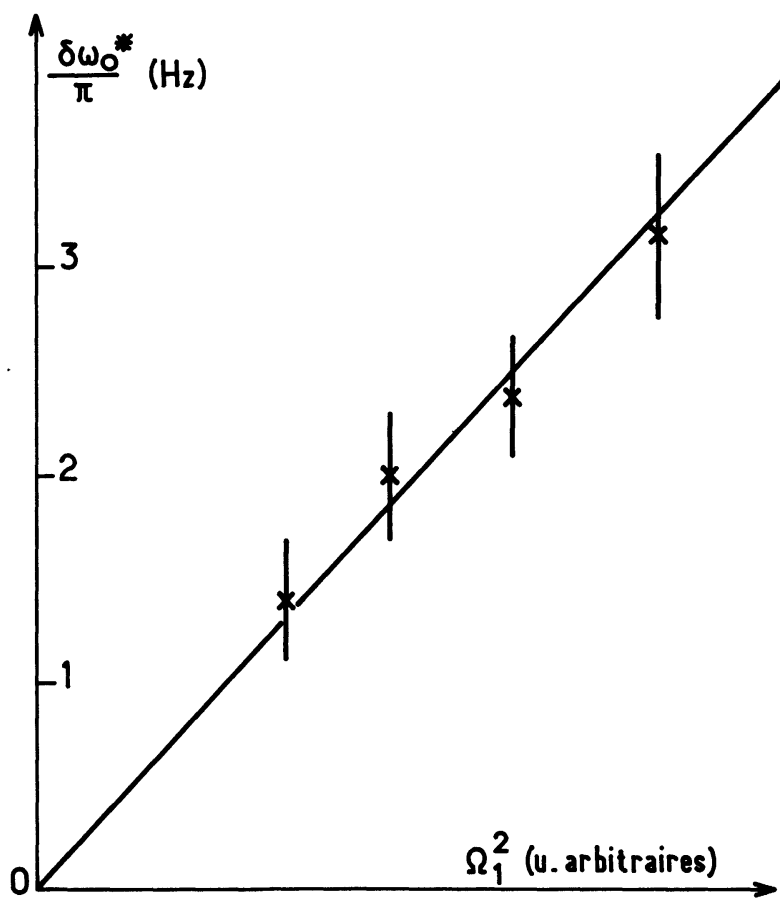

Fig. 17. - Valeurs mesurées pour le déplacement de fréquence $2 \delta \omega_{0}^{*} / 2 \pi$ en fonction du carré de l'intensité du champ RF inhomogène.

Nous avons porté sur la figure 18 les valeurs du produit $\delta \omega_{0}^{*} T_{1}^{*}$ en fonction de $\Delta$. On voit que les résultats se situent sensiblement sur une droite passant par l'origine, conformément à la formule (B.39b). La pente de la droite est, d'après cette formule, égale au temps de diffusion. Nous avons tracé, à titre de comparaison, une droite en pointillés dont la pente a la valeur de $\tau_{11}$ mesurée très précisément par ailleurs (voir $§ 2.2 .1$ ). On voit que cette droite en pointillés passe bien à l'intérieur des barres d'erreur de la plupart des points expérimentaux de la figure 18 . On trouve donc un bon accord quantitatif entre les prévisions théoriques et les mesures du déplacement de fréquence $\delta \omega_{0}^{*}$.

3.2 CAS OÙ LES CHAMPS STATIQUE ET DE RADIOFRÉQUENCE SONT TOUS DEUX INHOMOGÈNES. - NouS avons vu dans la partie théorique ( $\S$ C.2 de [1]) que, lorsque les inhomogénéités de champ comprennent une partie statique en plus d'une partie modulée, des effets nouveaux intéressants sont prévisibles en ce qui concerne la courbe étroite (forme de courbe, position). Ces effets sont liés aux termes non diagonaux de la matrice $(R)$ de relaxation, c'est-à-dire au caractère anisotrope de cette relaxation; nous avons d'ailleurs noté dans [1] que cette anisotropie est le résultat des corrélations entre les inhomogénéités des champs statique et oscillant. Au $\S \mathrm{C} .2 c-\gamma$, nous en avons donné une interprétation géométrique dans un cas simple.

Pour illustrer ces prévisions expérimentalement, nous avons choisi un dispositif de champ oscillant

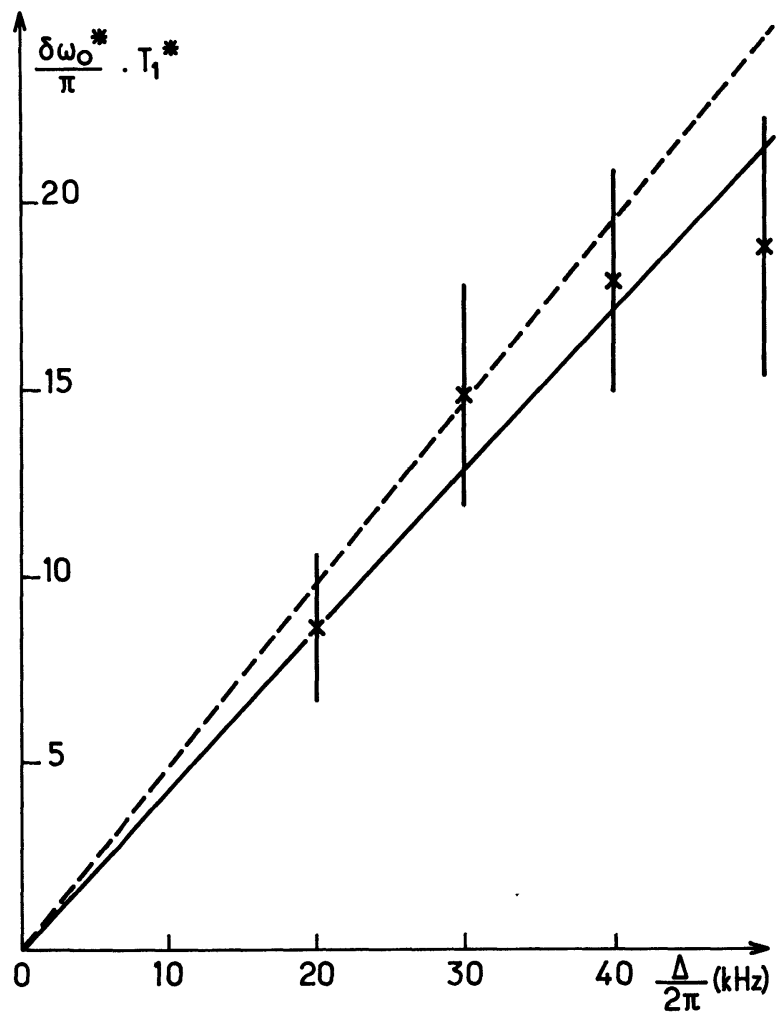

Fig. 18. - Valeurs expérimentales du produit $2 \frac{\delta \omega_{0}^{*}}{2 \pi} T_{1}^{*}$.

$\frac{\delta \omega_{0}^{*}}{2 \pi}$ : déplacement de la fréquence de résonance en présence du champ RF inhomogène ; $T_{1}^{*}$ : temps de relaxation longitudinal pour la valeur $-\omega^{\prime} / \gamma$ du champ statique $; \Delta=\omega^{\prime}-\omega\left(\omega^{\prime}\right.$ et $\omega$ : pulsations des champs RF homogène et inhomogène). La droite en pointillés a une pente calculée théoriquement (voir le texte).

inhomogène correspondant à ce cas particulier. La simplification introduite provient du fait que le champ oscillant ne possède pas de composante sur une direction $O y$ perpendiculaire à $O z$. Nous avons réalisé cette situation à l'aide d'un ensemble de 2 fils parallèles à $O y$ et passant de part et d'autre de la cellule. Ils sont alimentés en phase par deux tensions alternatives d'intensités comparables et réglables de façon à annuler toute partie homogène du champ RF sur la cellule. La configuration de champ oscillant est celle de la figure $1 c$. Les inhomogénéités du champ statique sont créées par une paire de bobines en Holtzhelm parallèles à celles qui fournissent le champ statique homogène $\boldsymbol{B}_{0}$. Les inhomogénéités de champs statique et RF sont alors bien corrélées. En effet, on montre en première approximation que, pour le champ statique créé par les bobines en Holtzhelm, on a $B_{0 z} \simeq \mu z$, tandis que pour le champ oscillant inhomogène produit par les deux fils :

$$
\left\{\begin{array}{l}
B_{1 x} \simeq \mu^{\prime} z \\
B_{1 z} \simeq \mu^{\prime} x
\end{array}\right.
$$

ce qui, dans le référentiel tournant, et pour un certain choix de l'axe $O X$, donne les inhomogénéités statiques 
suivantes :

$$
\left\{\begin{array}{l}
\Omega_{1 Z} \simeq-\frac{\mu Z}{\gamma} \\
\Omega_{1 X} \simeq-\frac{\mu^{\prime} Z}{2 \gamma} \\
\Omega_{1 Y}=0 .
\end{array}\right.
$$

Les composantes sur les axes $O Z$ et $O X$ des inhomogénéités de champ sont alors proportionnelles l'une à l'autre (corrélation totale). Le coefficient non diagonal $A_{X Z}$ de la matrice de relaxation $(R)$ est ainsi maximal et l'inégalité (C.12) de [1] devient simplement :

$$
A_{X Z}^{2}=A_{X X} \cdot A_{Z Z}
$$

Si l'on se reporte à l'interprétation géométrique du $\S(\mathrm{C} .2 c-\gamma)$ de [1], on voit que la direction $O \mathbf{u}$ de nonrelaxation (voir Fig. 6 de [1]) fait avec $O X$ l'angle $\theta$ tel que :

$$
\operatorname{tg} \theta=\frac{\Omega_{1 Z}}{\Omega_{1 X}}=\frac{2 \mu}{\mu^{\prime}} .
$$

Expérimentalement, on peut faire varier cet angle $\theta$ en jouant sur les tensions appliquées respectivement aux bobines de Holtzhelm et aux deux fils (paramètres $\mu$ et $\mu^{\prime}$ de (12)).

Enfin, nous avons ajouté à ce dispositif de champs inhomogènes une paire de petites bobines en Helmholtz d'axe perpendiculaire à $O z$, de façon à pouvoir appliquer éventuellement un champ de radiofréquence homogène $\left(\omega_{1} \neq 0\right)$ à la cellule. Ce champ RF homogène, de même fréquence que le champ RF créé par les 2 fils, a d'abord été pris en phase avec lui (les 2 champs sont portés par le même axe $O X$ dans le référentiel tournant). Un système de déphasage a permis, dans un deuxième temps, d'effectuer dans le référentiel tournant une rotation de l'un des champs par rapport à l'autre.

Nous distinguerons, dans la présentation des résultats expérimentaux, les cas où $\omega_{1}=0$ et où $\omega_{1} \neq 0$.

3.2.1 Sans champ de radiofréquence homogène $\left(\omega_{1}=0\right)$. - Nous avons, dans ces conditions, enre-

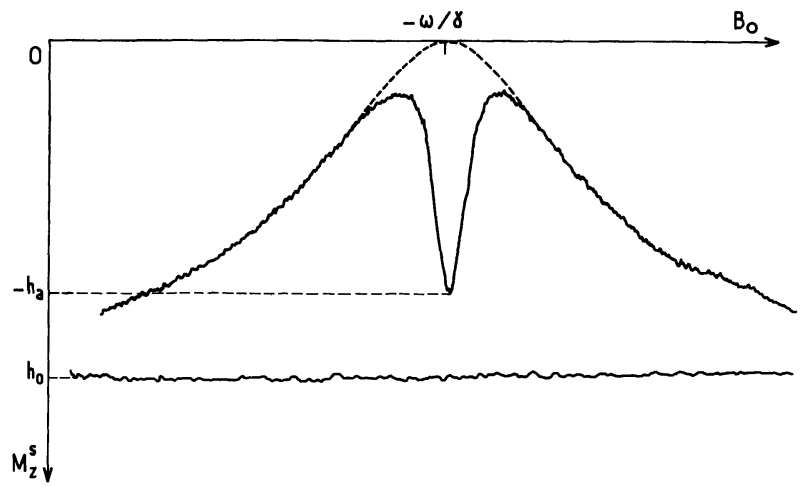

FIG. 19. - Courbe montrant l'existence d'un trou au centre de la courbe large (réorientation à résonance) lorsque le champ statique présente des inhomogénéités corrélées spatialement à celles du champ de radiofréquence. gistré des courbes de résonance nucléaire de ${ }^{3} \mathrm{He}$, qui ont l'allure de celle de la figure 19. On voit que, conformément aux prévisions de [1] $(\S \mathrm{C} .2 c-\alpha)$, une courbe fine apparaît au centre de la courbe large, bien qu'aucun champ de radiofréquence homogène ne soit appliqué à la cellule. De plus cette courbe fine est bien renversée (elle correspond à une réorientation des spins nucléaires à résonance, exactement à l'opposé de ce qui est observé en résonance magnétique habituelle). Sur la figure 20 , on voit l'apparition de la courbe fine renversée lorsqu'on applique un gradient de champ statique d'intensité croissante (la tension RF appliquée aux deux fils restant constante) : la hauteur $\left|h_{\mathrm{a}}\right|$ du trou augmente, ainsi que sa largeur $\mathcal{L}$. Ceci est en accord avec les formules (C.22a) et (C.22b) de [1], simplifiées grâce à (11) et au fait qu'il n'y a pas d'inhomogénéités de champ suivant l'axe $O Y$.

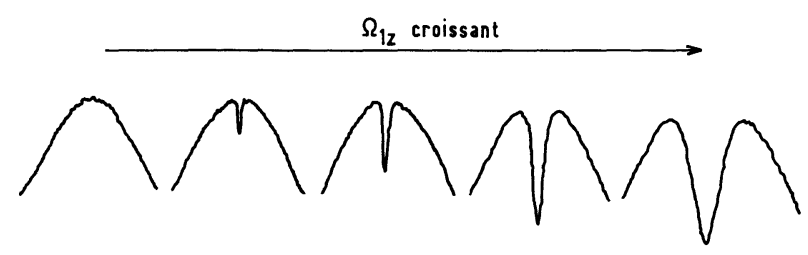

FIG. 20. - Courbes de résonance magnétique nucléaire de ${ }^{3} \mathrm{He}$ avec un champ de radiofréquence et un champ statique présentant des inhomogénéités corrélées (cas où la partie homogène du champ RF est nulle). Apparition du trou au sommet de la courbe large lorsque le gradient de champ statique croît en intensité à partir de zéro.

Nous avons cherché à vérifier quantitativement les prévisions théoriques. Nous nous sommes placés dans le cas simple où la courbe large est très saturée, c'est-à-dire où $A_{X X} \gg 1 / T_{1}^{0}$; compte tenu de cette hypothèse, de la nullité de la composante sur $O Y$ des inhomogénéités et de la corrélation totale entre ses composantes sur $O X$ et $O Z$ exprimée par (11), la formule (C.22a) de [1] devient simplement :

$$
\frac{\left|h_{\mathrm{a}}\right|}{h_{\mathrm{o}}}=\frac{A_{Z Z}}{A_{Z Z}+\frac{T_{1}^{0}}{T_{2}^{0}} A_{X X}}
$$

où $h_{\mathrm{o}}$ est la hauteur du signal d'orientation observé en l'absence de tout gradient de champ $\left(h_{\mathrm{o}}=\frac{M_{0} T_{1}^{0}}{T_{p}}\right.$ avec les notations de [1]). On peut encore mettre (13) sous la forme :

$$
\frac{h_{\mathrm{o}}}{\left|h_{\mathrm{a}}\right|}-1=\frac{T_{1}^{0}}{T_{2}^{0}} \frac{A_{X X}}{A_{Z Z}}=\frac{T_{1}^{0}}{T_{2}^{0}} \frac{1}{\operatorname{tg}^{2} \theta} .
$$

Pour vérifier cette loi expérimentalement, nous avons fait varier l'intensité $i_{z}$ du courant dans les bobines de Holtzhelm créant le gradient de champ statique, à courant fixe dans les fils créant le champ oscillant inhomogène (ainsi $\operatorname{tg} \theta \propto i_{z}$ ). Nous avons, pour chaque valeur de $i_{z}$, mesuré la hauteur $\left|h_{\mathrm{a}}\right| \mathrm{du}$ trou et la hauteur $h_{\mathrm{o}}$ de la courbe large saturée. Les 
résultats sont portés sur la figure 21 . On voit que les valeurs de $\frac{h_{\mathrm{o}}}{\left|h_{\mathrm{a}}\right|}-1$, portées en fonction de $1 / i_{z}^{2}$, s'alignent sensiblement sur une droite passant par l'origine, ce qui est en bon accord avec les prévisions de (14).

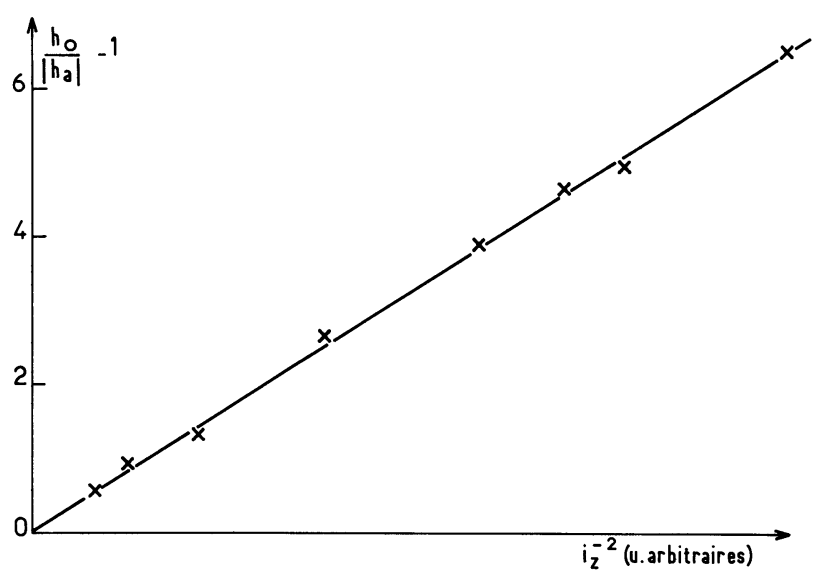

FIG. 21. - Variations de l'amplitude relative $\left|h_{\mathrm{a}}\right| / h_{\mathrm{o}}$ du trou central de la figure 19, en fonction du carré de l'intensité $i_{z}$ du courant dans les bobines créant le gradient de champ statique.

Des vérifications analogues ont été faites dans le cas où la courbe large est désaturée. Nous avons également étudié quantitativement le comportement de la largeur $\mathfrak{L}$ du trou. Il serait trop long de donner ici le détail de ces expériences. Signalons que, dans tous les cas, un bon accord a été constaté avec les formules (C.22) de [1].

3.2.2 Avec champ de radiofréquence homogène $\left(\omega_{1} \neq 0\right)$. - La figure 22 montre comment la courbe large avec un trou central du § précédent se trouve

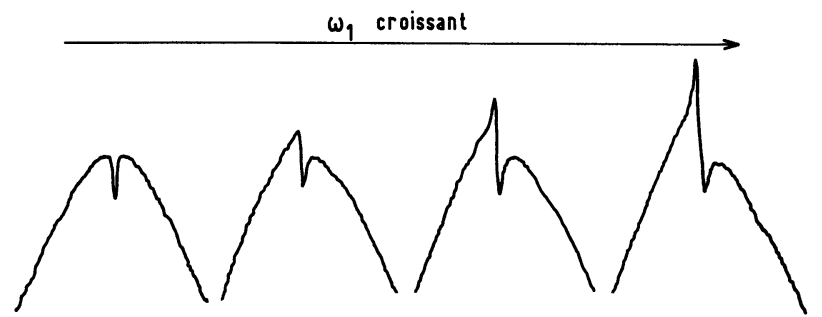

Fig. 22. - Courbes de RMN de ${ }^{3} \mathrm{He}$ avec un champ de radiofréquence et un champ statique présentant des inhomogénéités corrélées; cas où le champ RF comporte une partie homogène $\omega_{1} / \gamma$. On note l'apparition d'une courbe de dispersion centrale au sommet de la courbe large.

modifiée lorsqu'on applique en outre un champ de radiofréquence homogène d'intensité $\omega_{1}$ croissante à la cellule. Conformément aux prévisions du $\S \mathrm{C} .2 c-\beta$ de [1], on voit apparaître une courbe de dispersion superposée à la courbe d'absorption centrale, l'amplitude de cette dernière semblant d'ailleurs décroître avec $\omega_{1}$. La courbe (2) de la figure 23 montre un exemple d'une telle courbe fine de forme complexe apparaissant au sommet de la courbe large. A titre de comparaison, nous avons tracé sur la même figure la courbe (1) enregistrée dans les mêmes conditions,

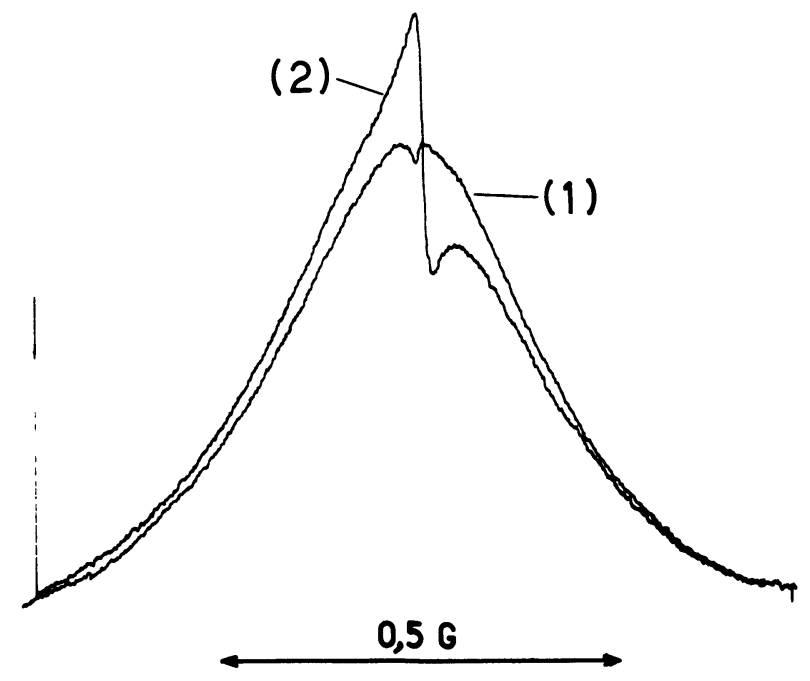

FIG. 23. - Courbes typiques montrant la disposition relative des courbes larges et étroites :1) avec $\omega_{1}=0 ; 2$ ) avec $\omega_{1} \neq 0$.

mais sans champ RF homogène. La partie centrale de ces courbes a été reportée avec une échelle dilatée en abscisses sur la figure 24. Sur cette figure, on voit nettement apparaître un autre phénomène : le déplacement $\delta \tilde{\omega}$ de la position de la courbe fine avec $\omega_{1}$.

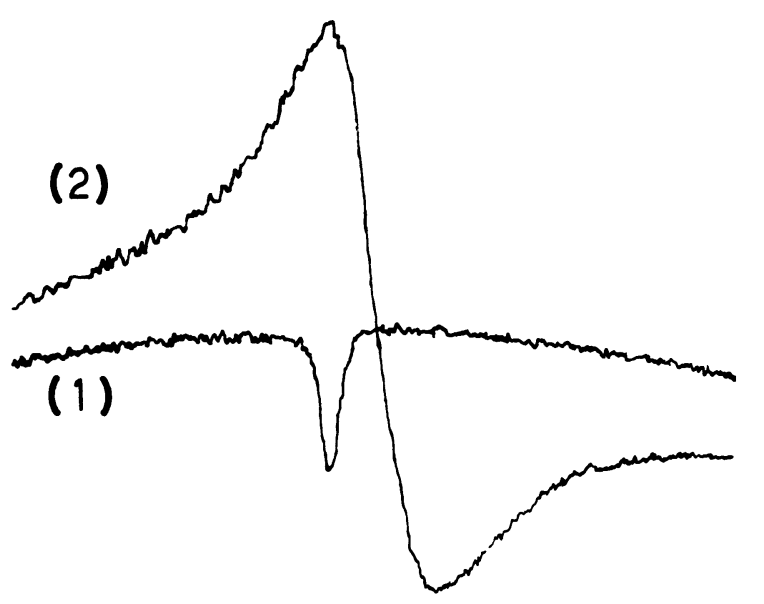

$20 \mathrm{mG}$

Fig. 24. - Agrandissement de la partie centrale de la figure 23 : 1) avec $\left.\omega_{1}=0 ; 2\right)$ avec $\omega_{1} \neq 0$. Le déplacement de fréquence $\delta \widetilde{\omega} / 2 \pi$ apparaît clairement sur la courbe (2).

Pour une étude systématique de ces effets, nous avons, dans chaque cas, dépouillé les enregistrements de ces courbes fines de façon à en tirer les 2 courbes d'absorption et de dispersion qui les constituent. Nous avons pour cela supposé, conformément à la théorie, qu'elles sont toutes deux centrées au même point et de même largeur. Connaissant la position de l'asymptote (par le sommet de la courbe large en l'absence de $\omega_{1}$ ), il est aisé de connaître, par une construction géométrique simple, la position de ce centre (voir par exemple l'appendice de la référence [14]). On en déduit alors immédiatement les 
courbes paire et impaire centrées en ce point dont la somme donne la courbe initiale. On vérifie bien que ces deux courbes de décomposition ont une forme, l'une d'absorption lorentzienne, l'autre de dispersion. On en tire alors simultanément une mesure du déplacement de fréquence $\delta \tilde{\omega} / 2 \pi$, de la hauteur $h_{\mathrm{a}}$ de la courbe d'absorption et de celle $h_{\mathrm{d}}$ de la courbe de dispersion, ainsi que de leur largeur commune $\mathcal{L}$. Notons que $h_{\mathrm{a}}$, qui est toujours négatif pour $\omega_{1}=0$ (cas du trou), peut soit devenir positif, soit rester négatif quand $\omega_{1}$ croît.

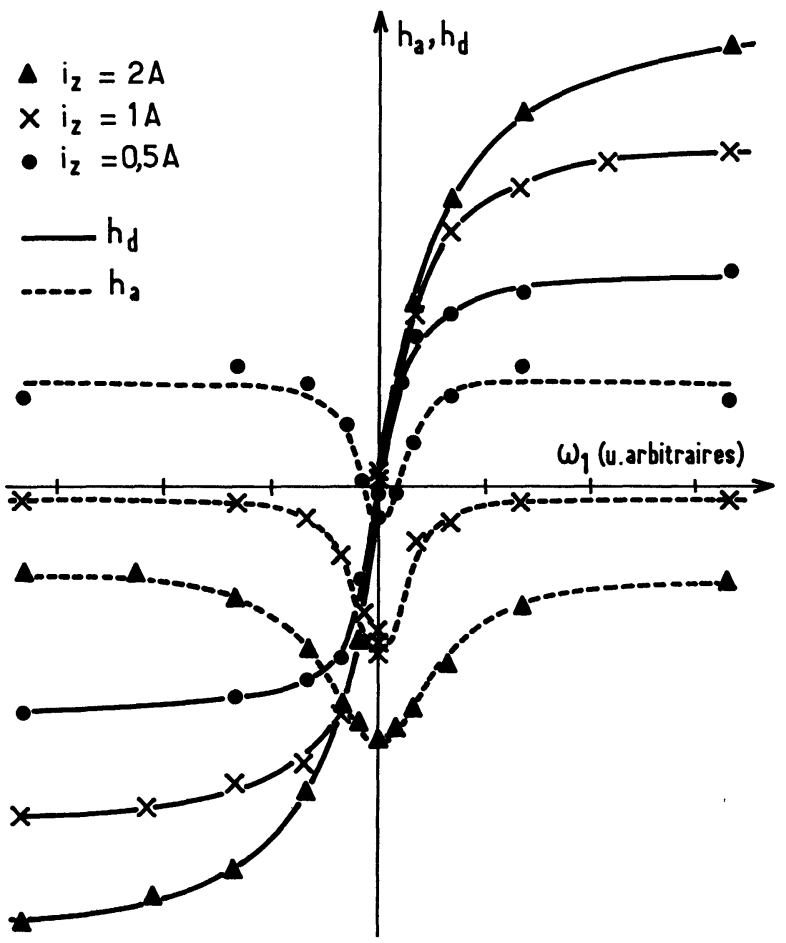

FIG. 25. - Variations avec $\omega_{1}$ des amplitudes $h_{\mathrm{a}}$ et $h_{\mathrm{d}}$ des courbes d'absorption et de dispersion centrales. Les 3 séries de courbes correspondent à des valeurs croissantes de l'intensité $i_{z}$ du courant produisant le gradient de champ statique.

Sur la figure 25 , nous avons porté trois séries de résultats relatifs aux hauteurs $h_{\mathrm{a}}$ et $h_{\mathrm{d}}$ en fonction de $\omega_{1}$. Chacune des 3 séries correspond à une valeur donnée de $i_{z}$ (proportionnel au gradient de champ statique). En ce qui concerne les hauteurs $h_{\mathrm{d}}$, comparons ces résultats à la formule (C.21) de [1]; à gradients de champ donnés, celle-ci prévoit que $h_{\mathrm{d}}$ est une fonction impaire de $\omega_{1}$ qui tend vers une constante si $\omega_{1} \rightarrow \infty$; en fait, c'est bien ce qu'on constate sur la figure 25. Par contre, d'après la formule (C.20) de [1], la hauteur $h_{\mathrm{a}}$ se comporte comme une courbe d'absorption en fonction de $\omega_{1}$ : ceci apparaît nettement sur la figure 25 . Les valeurs de $i_{z}$ ont été choisies de façon à illustrer les trois situations possibles pour la valeur limite de $h_{\mathrm{a}}$ aux fortes valeurs de $\omega_{1}$ (cf. formule (C.24) de [1]) : cette valeur limite reste négative si les gradients de champ sont très forts et corrélés $\left(A_{X Z}^{2}>\frac{1}{2} \Gamma_{X} \Gamma_{Z}\right)$; elle peut être nulle $\left(A_{X Z}^{2}=\frac{1}{2} \Gamma_{X} \Gamma_{Z}\right)$, cas auquel la courbe de dispersion apparaît seule aux fortes valeurs de $\omega_{1}$; elle peut enfin être positive
$\left(A_{X Z}^{2}<\frac{1}{2} \Gamma_{X} \Gamma_{Z}\right)$. Notons que, conformément aux formules (C.20) et (C.21) de [1], la largeur des courbes de la figure 25 augmente avec l'intensité du gradient de champ statique.

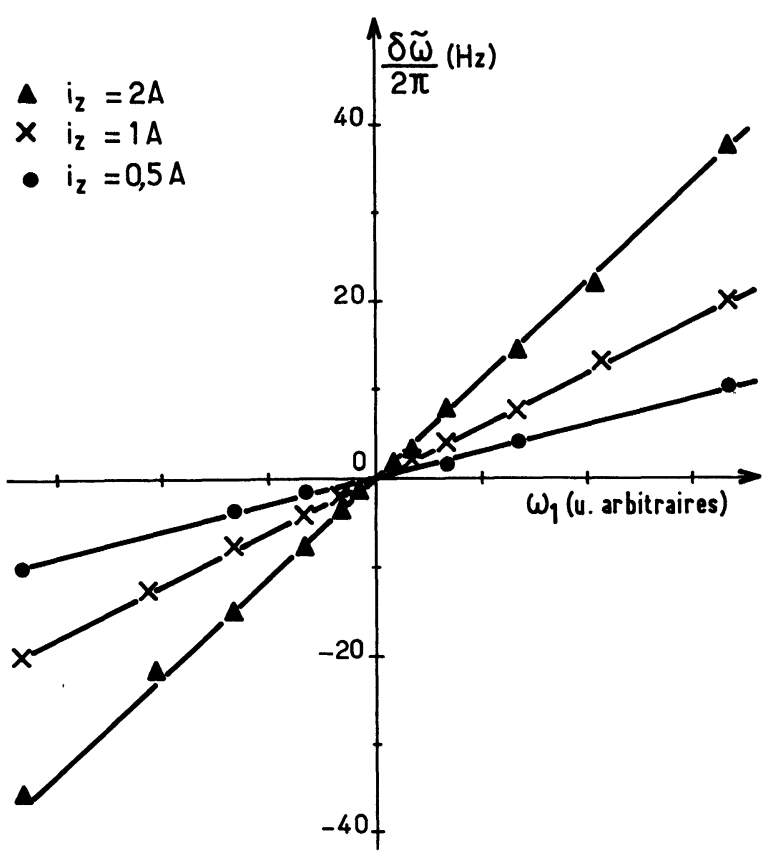

FIG. 26. - Variations avec $\omega_{1}$ du déplacement $\delta \tilde{\omega} / 2 \pi$ de la fréquence de résonance pour les mêmes valeurs de $i_{z}$ (gradient de champ statique) que sur la figure 25 .

Nous avons porté sur la figure 26 les déplacements $\delta \tilde{\omega} / 2 \pi$ du centre de la résonance mesurés pour la même série d'enregistrements. On constate bien qu'à intensité donnée des gradients de champ, $\delta \tilde{\omega} / 2 \pi$ varie linéairement avec $\omega_{1}$. Ceci est conforme à la formule (C.18) de [1] $\left({ }^{3}\right)$. En particulier, si la phase du champ RF homogène est changée de $\pi$ par rapport à celle du champ RF inhomogène ( $\omega_{1}$ est changé en - $\left.\omega_{1}\right)$, le déplacement $\delta \tilde{\omega} / 2 \pi$ est bien changé de signe. De plus, à valeur de $\omega_{1}$ fixe, $\delta \tilde{\omega} / 2 \pi$ varie proportionnellement à l'intensité du gradient de champ statique (cf. (C.18) de [1] qui montre que $\delta \tilde{\omega} \propto A_{X Z}$ ). On note que ces déplacements de fréquence peuvent être du même ordre de grandeur que la largeur $\mathcal{L}$ des courbes d'absorption et de dispersion précédentes. Sur la figure 27, nous avons porté avec la même échelle les valeurs de $\left[\right.$ et de $\delta \widetilde{\omega} / 2 \pi$ en fonction de $\omega_{1}$ pour une même série de courbes. Le déplacement de la position de la résonance sous l'effet des gradients de champ n'est donc pas du tout négligeable dans cette expérience.

Enfin nous avons fait varier la différence de phase $\varphi$ entre les champs RF inhomogène et homogène. Ceci équivaut à faire tourner d'un angle $\varphi$ l'ellipsoïde de relaxation par rapport à la direction qui porte la composante tournante du champ de radiofréquence

$\left({ }^{3}\right)$ Note ajoutée aux épreuves : signalons qu'une erreur s'est introduite dans l'expression simplifiée de $\delta \tilde{\omega}$ donnée dans la formule (4) de [3], qu'il faut lire : $\delta \tilde{\omega}=\omega_{1} T_{1} \Omega_{z x}^{2} \tau_{\mathrm{d}} / 2$. 


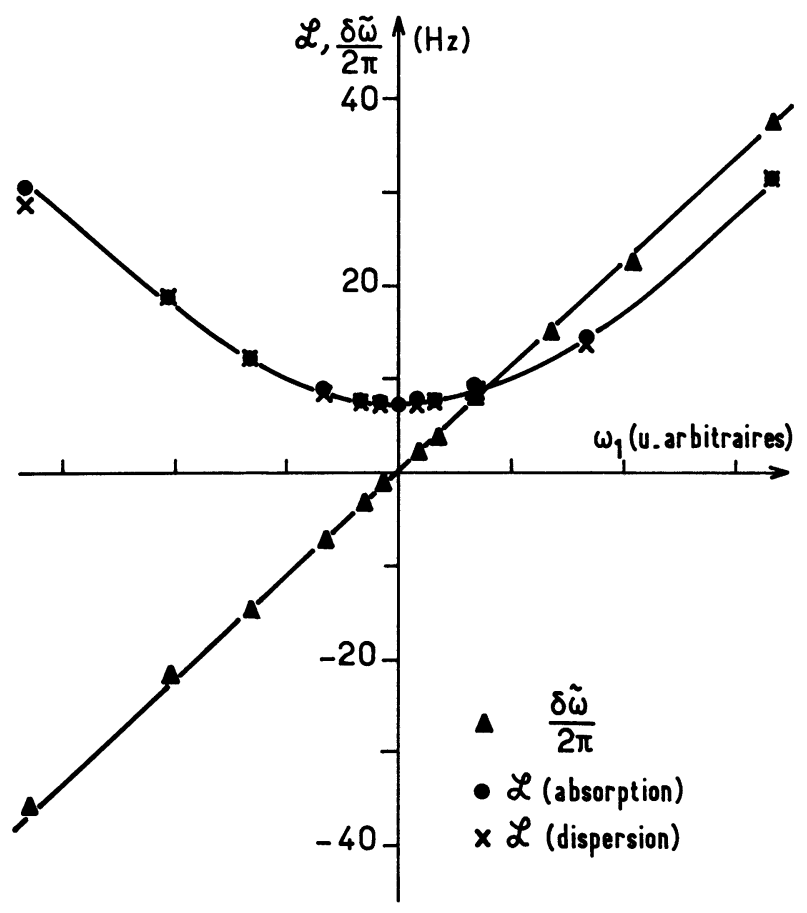

Fig. 27. - Variations avec $\omega_{1}$ de la largeur $\mathcal{L}$ et du déplacement $\delta \tilde{\omega} / 2 \pi$ des courbes de dispersion et d'absorption centrales.

homogène. Clairement, ceci indique que le paramètre $A_{X Z}$ varie comme $\cos \varphi$. Il en est de même pour l'amplitude $h_{\mathrm{d}}$ de la courbe de dispersion et pour le déplacement $\delta \tilde{\omega}$. La vérification expérimentale confirme bien la loi $h_{\mathrm{d}} \propto \cos \varphi$. En particulier lorsque $\varphi=90^{\circ}$ (les deux champs RF sont en quadrature; $A_{X Z}=0$ ), seule une courbe d'absorption non déplacée apparaît. Son comportement en fonction de $\omega_{1}$ est conforme à la formule (C.20) de [1] (avec $A_{X Z}=0$, mais $A_{Y Z} \neq 0$ ) et du même type que les variations de $h_{\mathrm{a}}$ indiquées sur la figure 25 .

4. Résonance à deux quanta. - 4.1 PRINCIPE DE L'EXPÉRIENCE. - Au $\S$ C. $2 d$ de [1], nous avons prévu l'existence d'une résonance à 2 quanta en champ de radiofréquence inhomogène (résonance centrée à la valeur $B_{0}=-2 \omega / \gamma$ du champ statique $B_{0}$, si $\omega$ est la pulsation du champ oscillant). D'après [1] ceci nécessite que le champ de radiofréquence présente des inhomogénéités corrélées pour ses composantes parallèles aux axes $O Z$ et $O X(O X$ : axe du référentiel tournant qui porte la composante transversale du champ de radiofréquence). En d'autres termes, avec les notations du $\S$ C. $2 d$ de [1], le produit scalaire

$$
\Omega_{1 z+} \propto\left\langle\Omega_{1 z} \mid \Omega_{1+}\right\rangle
$$

doit être non nul sur le volume de la cellule. Nous avons réalisé cette condition expérimentalement en créant le champ RF à l'aide d'une paire de bobines connectées en Holtzhelm, et dont l'axe horizontal fait l'angle $\alpha$ avec l'axe $O z$. On montre que dans ce cas :

$$
\Omega_{1 z+} \propto \Omega_{1}^{2} \sin 2 \alpha
$$

où $\Omega_{1}$ est une quantité proportionnelle à l'intensité du champ inhomogène donné par les bobines. Dans un premier temps, de façon à produire un effet maximal, nous avons choisi la valeur $\alpha=45^{\circ}$.

L'intensité de la résonance à 2 quanta est donnée par la formule (C. 37) de [1] (notons que les temps $T_{1}$ et $T_{2}$ qui y figurent sont les temps de relaxation globale à la valeur $-2 \omega / \gamma$ du champ statique, compte tenu de la relaxation longitudinale et transversale additionnelles introduites par les inhomogénéités du champ oscillant).

La valeur des paramètres expérimentaux $\omega$ et $\Omega_{1}$ a été choisie pour réaliser un compromis entre deux exigences contradictoires : d'une part, à $\omega$ donné, on a intérêt à avoir $\Omega_{1}$ grand (la résonance à deux quanta croît comme $\Omega_{1}^{4}$ tant qu'elle n'est pas saturée); d'autre part, il ne faut pas que $\Omega_{1}$ soit si intense que le temps de relaxation $T_{1}$ en soit trop raccourci au voisinage de la résonance à deux quanta : dans ce cas, celle-ci ne serait pas visible car il ne lui resterait plus d'orientation atomique à détruire. On voit donc qu'on a intérêt à se placer en champ assez fort. En pratique, nous avons été limités par la puissance de radiofréquence disponible. Ceci nous a conduits à choisir pour $\omega / 2 \pi$ la valeur de $32 \mathrm{kHz}$, ce qui laisse prévoir une résonance à deux quanta au voisinage d'un champ $B_{0}$ de 20 Gauss. Signalons que nous avons réalisé un accord résonnant des bobines de radiofréquence, pour une fréquence très voisine de $32 \mathrm{kHz}$, de façon à diminuer considérablement l'harmonique à la fréquence double, susceptible de produire une résonance normale mais ici parasite à la fréquence $2 \omega / 2 \pi$. Au cours de toutes ces expériences, nous avons d'ailleurs contrôlé constamment, à l'aide d'un analyseur d'ondes, le taux d'harmonique 2 présent dans la tension aux bornes des bobines.

4.2 MiSE EN ÉVIDENCE. VÉRIFICATIONS QUANTITATIVES. - A l'aide du montage précédemment décrit, nous avons d'abord procédé à l'annulation soigneuse de la partie homogène $\omega_{1}$ du champ de radiofréquence à la fréquence $\omega / 2 \pi$, en faisant disparaître totalement la courbe étroite au sommet de la courbe large centrée en $B_{0}=-\omega / \gamma$. Le but de ce réglage très critique est d'éviter de produire au champ $B_{0}=-2 \omega / \gamma$ la résonance à deux quanta habituelle que l'on observe d'ordinaire avec un champ de radiofréquence homogène.

Puis, en balayant le champ $B_{0}$ au voisinage de la valeur $-2 \omega / \gamma$, nous avons enregistré des courbes analogues à celles de la figure 28. Elles correspondent à des valeurs croissantes du paramètre $\Omega_{1}$. On voit qu'elles apparaissent sur un fond continu d'autant plus important que $\Omega_{1}$ est grand (aile de la courbe large centrée en champ moitié). Nous avons identifié ces résonances à deux quanta comme liées à la présence des inhomogénéités du champ RF.

Ceci a été confirmé par les vérifications quantitatives suivantes. Avec les notations indiquées sur la figure 28, 


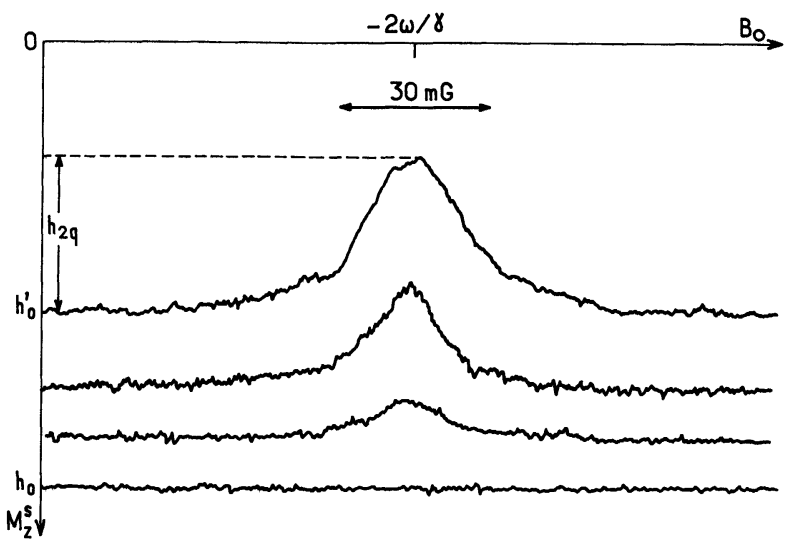

FIG. 28. - Enregistrement de résonances à 2 quanta en champ de radiofréquence totalement inhomogène. Les différentes courbes correspondent à des valeurs croissantes de l'intensité du champ RF inhomogène. $p=1$ torr $; \omega / 2 \pi=32 \mathrm{kHz}$.

et en utilisant (15), on peut récrire l'équation (C.37) de [1] sous la forme :

$$
\frac{h_{0}^{\prime}}{h_{2 \mathrm{q}}}-1 \propto \frac{4 \omega^{2}}{T_{1} T_{2}} \times \frac{1}{\Omega_{1}^{4}} \times \frac{1}{\sin ^{2} 2 \alpha} .
$$

Nous avons d'abord étudié le comportement de la hauteur $h_{2 q}$ de la résonance à deux quanta en fonction de l'intensité $\Omega_{1}$ du champ RF inhomogène. Remarquons que dans (16) $T_{1}$ est une quantité qui peut aussi dépendre de $\Omega_{1}$, comme l'attestent les variations du fond continu des résonances de la figure 28 . Nous avons donc, pour chaque valeur de $\Omega_{1}$, enregistré simultanément la résonance à deux quanta et une transitoire de pompage donnant une mesure directe

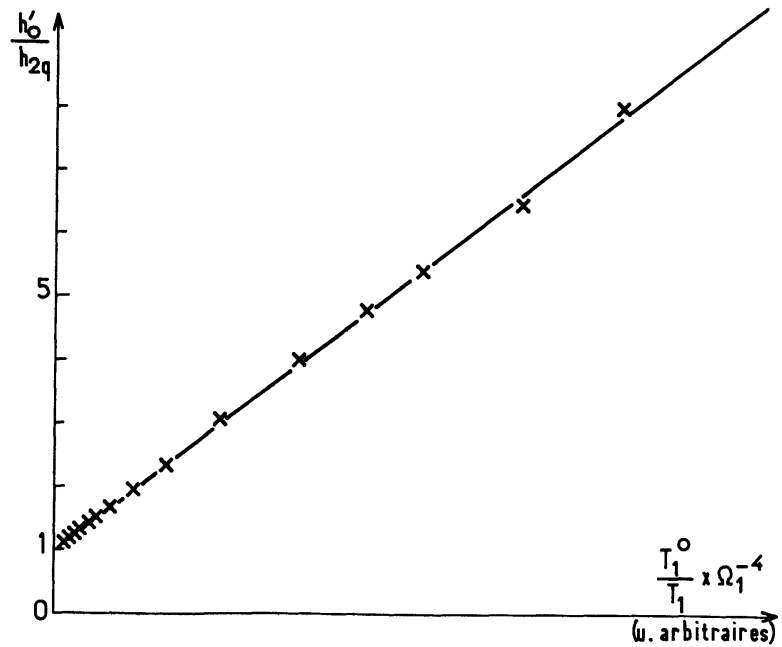

FIG. 29. - Variation de l'amplitude de la résonance à deux quanta en fonction de l'intensité du chámp RF inhomogène.

$h_{2 \mathrm{q}} / h_{\mathrm{o}}^{\prime}$ : amplitude relative de la résonance à 2 quanta.

$\Omega_{1} / \gamma$ : intensité du champ RF inhomogène.

$T_{1}$ : temps de relaxation longitudinal global à la valeur $-2 \omega /$; du champ statique $B_{0}$.

$T_{1}^{0}$ : temps de relaxation longitudinal en l'absence de champ RF inhomogène. de $T_{1}$ à la valeur $-2 \omega / \gamma$ du champ $B_{0}$. Nous avons porté sur la figure 29 les valeurs de $h_{0}^{\prime} / h_{2 q}$ trouvées en fonction de $1 / T_{1} \Omega_{1}^{4}$. On constate que les points expérimentaux se répartissent très exactement sur une droite dont l'ordonnée à l'origine est égale à 1 . Ceci est en excellent accord avec la formule (16).

De plus, nous avons mis en évidence la dépendance en $\alpha$ de la hauteur $h_{2 q}$. Nous avons montré que la résonance à 2 quanta disparaît pratiquement totalement lorsque $\alpha=90^{\circ}$ et $\alpha=0^{\circ}$. Pour des valeurs intermédiaires de $\alpha$, nous avons tenu compte des variations éventuelles de $T_{1}$ en mesurant simultanément $h_{0}, h_{0}^{\prime}$ et $h_{2 q}$ (voir Fig. 28). (On sait que $h_{0} / h_{0}^{\prime}=T_{1}^{0} / T_{1}$.) Ces résultats sont portés sur la figure 30. Ils présentent un accord acceptable avec la formule (16), compte tenu des importantes barres d'erreur affectant les mesures.

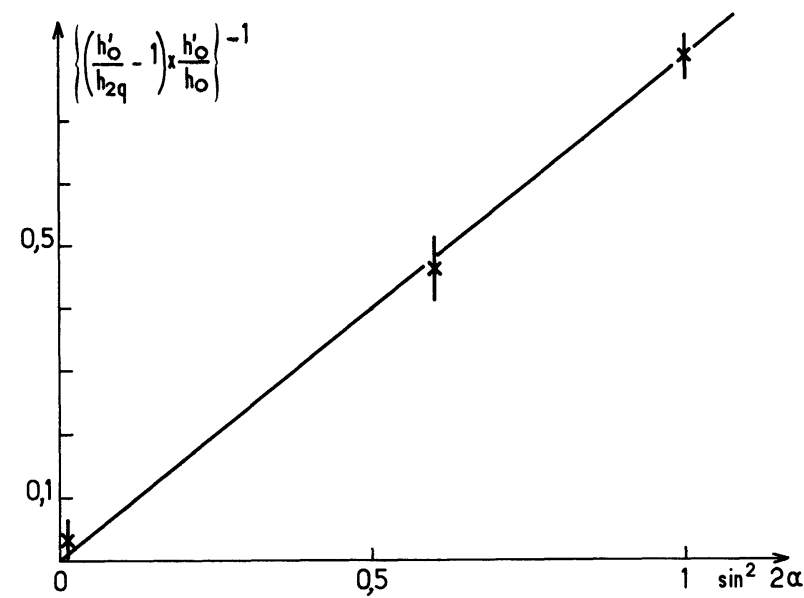

FIG. 30. - Comportement de l'amplitude de la résonance à 2 quanta en fonction de l'orientation des bobines produisant le champ RF inhomogène.

Conclusion. - Nous avons décrit dans cet article un ensemble d'expériences de résonance magnétique nucléaire de ${ }^{3} \mathrm{He}$ effectuées dans des conditions assez variées de champ de radiofréquence inhomogène. Les résultats expérimentaux vérifient dans le détail toutes les prévisions théoriques exposées dans un article précédent. Parmi tous les effets nouveaux qui ont été mis en évidence, deux surtout retiennent l'attention, car ils sont susceptibles d'avoir des conséquences pratiques.

D'une part, lorsque le mouvement des spins est régi par un processus de diffusion, l'étude de la résonance magnétique en champ de radiofréquence très inhomogène fournit une méthode de mesure du coefficient de diffusion du gaz.

D'autre part, lorsque le champ statique présente également des inhomogénéités corrélées à celles du champ de radiofréquence, d'importants déplacements de la position de la résonance sont possibles. Dans les mesures de très haute précision, cette cause d'erreur systématique n'est pas toujours à négliger. 


\section{Bibliographie}

[1] Barbé, R., Leduc, M. et Lalö̈, F., J. Physique 35 (1974) 699

[2] Brossel, J., "Atomic Physics 3 ", Proceedings of the Third International Conference on Atomic Physics, Boulder 1972, p. 435 (Plenum Press).

[3] Barbé, R., LeduC, M. et Lalö̈, F., Nuovo Cimento Lett. 8 (1973) 915

[4] Colegrove, F. D., Schearer, L. D. et Walters, G. K., Phy's. Rev. 132 (1963) 2561.

[5] Schearer, L. D., Thèse, Rice University (1966).

[6] Dupont-Roc, J., Leduc, M. et Lalö̈. F., J. Phlvique 34 (1973) 961 et 977.
[7] Pavlovic, M. et Lalö̈, F., J. Physique 31 (1970) 173.

LALOË, F., Thèse, Paris (1970) : Annls. de Phys. 6 (1971) 5.

[8] Du Bro, G. A. et Weissman, S., Phys. Fluids 13 (1970) 2682.

[9] Bendt, P. J., Phys. Rev. 110 (1958) 85.

[10] Hahn, E. L., Phys. Rev. 80 (1950) 580.

[11] Abragam, A., Les principes du magnétisme nucléaire (P.U.F.) 1961.

[12] Novikov, L. N., Votiakov, S. et PoutiRsky, V., C. R. Hebd. Séan. Acad. Sci. 277B (1973) 175.

[13] Popescu, I. M. et Novikov, L. N., C. R. Hebd. Séan. Acad. Sci. 259 (1964) 1321.

[14] Dupont-Roc, J., Thèse de 3e cycle, Paris (1968). 